\title{
Article
}

Mycosphere

Doi 10.5943/mycosphere/7/3/1

Copyright () Guizhou Academy of Agricultural Sciences

\section{Biodiversity and molecular characterization of yeast and filamentous fungi in the air of citrus and grapevine plantations in Assiut area, Egypt}

\author{
Moubasher AH*, Abdel-Sater MA and Zeinab Soliman \\ Department of Botany and Microbiology, Faculty of Science, Assiut University, P.O. Box 71516, Assiut, Egypt
}

Moubasher AH, Abdel-Sater MA, Soliman Zeinab SM 2016 - Biodiversity and molecular characterization of yeast and filamentous fungi in the air of citrus and grapevine plantations in Assiut area, Egypt. Mycosphere 7(3), 236-261, Doi 10.5943/mycosphere/7/3/1

\begin{abstract}
A total of 218 species and 3 varieties belonging to 83 genera of filamentous and yeast fungi were recovered from the air of both citrus and grapevine plantations. A relatively higher numbers of genera and species were recovered from the air of citrus plantations compared with those recovered from grapevine plantations. The peak of total propagules of fungi caught from the air of citrus plantations was shown in February on both media and from the air of grapevine in December and August on DYM and DRBC, respectively. Their troughs were shown in June and October on DYM and DRBC, respectively for both citrus and grapevine plantations. The widest spectrum of species recovered from the air of citrus plantations was registered in June on both media and from the air of grapevine plantations in February and in April on DYM and DRBC, respectively. The air of citrus plantations shared the air of grapevine plantations in some highly encountered filamentous fungi on both media (Cladosporium, Penicillium, Aspergillus and Alternaria) or on one medium (Cochliobolus, Fusarium, Myrothecium, Phoma and Pleospora). Eighty-four fungal species were isolated from the air of citrus only, while 46 species were isolated from the air of grapevine only.Yeast fungi showed their peak of total propagules from the air of citrus plantations in October and April and from the air of grapevine plantations in June and December on DYM and DRBC, respectively. Fifteen genera and 26 species of yeasts were collected. Two genera of yeasts were encountered in high frequency on one medium and moderate or low on the other medium in the air of both citrus and grapevine plantations and these were Cryptoccocus (4 species) and Rhodotorula (3 species).
\end{abstract}

Key Words - Aerobiota - characterization - genotypic - phenotypic - seasonal fluctuation

\section{Introduction}

In the atmosphere many microbioparticles are present. These are fungal spores, pollen grains, insect parts. The study of aeromycology is important in plant pathology and in disease forecasting of plant diseases (Gregory 1973). Many fungal diseases of plants are spread by air. The deterioration of stored materials and the spoilage of foodstuffs are induced by growth of fungi which reach them from the air. Some fungal spores are regarded as important causes of allergic diseases such as bronchial asthma and allergic rhinitis. The fungal spores act passively as allergens acting on some individuals who have become sensitized (Moubasher 1993). 
Aerobiological studies conducted in relation to respiratory allergic diseases (Hasnain et al. 1984, 1985a,b) revealed that the total air spora of the Auckland region and many of its fungal components show a strong tendency towards an increase during warmer months and a gradual decline during winter. It is well known that fungi require certain optimum conditions for each phase of their growth (Gregory 1973). Aeromycological researchs from the Middle East area are scattered, in Egypt (Moubasher \& Moustafa 1974, Abu-El-Souod 1974, Abdel-Hafez et al.1986, Abdul Wahid et al.1996, Ismail et al. 2002), in Kuwait (Moustafa 1975, Khan et al. 1999), in Qatar (Al-Subai 2002), in Saudi Arabia (Abdel-Hafez 1984, Hasnain et al. 2005), in Yemen (El-Essawy et al. 1992), in Turkey (Sarica et al. 2002; Asan et al. 2004, Özkara et al. 2007), in Iran (Hedayati et al. 2005, Nourian et al. 2007) and in Jordan (Shaheen 1992, Al-Qura'n 2008).

Ben-Meir-Glueck (1952) isolated more than thirty different species from the air of orange groves and packing sheds and from the skins of fruits. These included Penicillium italicum and $P$. digitatum, which are the main incitants of citrus rot. Barkai-Gollan (1961) studied the air-borne fungi in citrus fruit packing houses and reported that $P$. digitatum and $P$. italicum predominated, whereas Fusarium, Trichoderma, Colletotrichum and Diplodia were encountered only occasiona1ly. Moubasher et al. (1971) found that the fungal aerospora in citrus plantations was considerably different from that in the soil with Cladosporium herbarum, Aspergillus niger, Curvularia sp., Alternaria alternata, Helminthosporium sativum and Fusarium were the most abundant in the air of citrus plantations. Fifteen isolates from air sampled at the vineyard were identified as $A$. niger (88.2\%) and 2 isolates were A. carbonarius (11.8\%) (Díaz et al. 2009). In the aerospora of tomato plantations Cladosporium sp. was found to be the most dominant type followed by species of Alternaria, aspergilli, Nigrospora, Torula and Curvularia (Lohare et al. 2009).

There are several reports on the occurrence of yeasts in the air (Di Menna 1955, Turner 1966, Gregory 1973). Al-Doory (1967) found that species of Cryptococcus, Rhodotorula, Sporobolomyces, and Debaryomyces were the most dominant species from the air in San Antonia, Texas, U.S.A. Rhodotorula mucilaginosa and Cryptococcus albidus were the most dominant species followed by Debaryomyces hansenii isolated from the air of El-Minia city, Egypt while Rhodotorula rubra, $R$. aurantiaca, Kluyveromyces marxianus, Torulaspora delbrueckii, Saccharomyces kluyveri, and Hansenula polymorpha were of less frequency (Haridy 1992).

The present work was designed to investigate the diversity of filamentous and yeast (for the first time in this laboratory) fungi in the air of two economically-important plants (citrus and grapevine). Also, seasonal fluctuations of these fungi were carried out bimonthly during April 2008 to February 2009.

\section{Materials \& Methods}

\section{Sampling location}

This study was carried out in Sahel-Saleem city at approximately $25 \mathrm{~km}$ south-east of Assuit city. Sampling was conducted bimonthly over a twelve - month period from April 2008February 2009. Three different plantations of citrus in the suburbs of Sahel-Saleem city and three of grapevine in El-Khawaled village (about $6 \mathrm{Km}$ to the east border of the river Nile), in the northeast of Sahel-Saleem city were selected.

\section{Isolation of air -borne fungi}

Five replicate plates of $9 \mathrm{~cm}$ diameter of each of two media (DYM and DRBC) were exposed for five minutes at a height of $60 \mathrm{~cm}$ above the ground level during the same hours of the day $(10 \mathrm{am}-2 \mathrm{pm})$ at each of the six sites. The plates were then sealed and brought back to the laboratory and incubated at $25^{\circ} \mathrm{C}$ for $7-15$ days, during which, the developing colonies were counted, isolated and identified. The meteorological data during the period of study were as follows: the maximum temperatures varied from $25^{\circ} \mathrm{C}-46^{\circ} \mathrm{C}$, the relative humidity from $36-86 \%$. A total of 36 exposures ( 3 farms of each of citrus and grapevine) were carried out bimonthly, with collectively 360 exposed plates. 


\section{Media used for isolation}

The exposure plate method relied on two isolation media "yeast extract malt extract agar supplemented with dichloran (DYM) and dichloran rose bengal chloramphenicol agar (DRBC)" were used in this study:

1- Dichloran yeast extract malt extract agar (DYM): Yeast extract malt extract agar (Wickerham 1951) of the following composition was employed: (g/l) yeast extract 3.0, malt extract 3.0, peptone 5.0, glucose 10.0, agar 20.0. Chloramphenicol $(250 \mu \mathrm{g} / \mathrm{ml})$ was used as a bacteriostatic agent. This medium was modified in the present work after preliminary survey by addition of $1 \mathrm{ml} / \mathrm{l}$ of $2 \mathrm{mg}$ of dichloran dissolved in $10 \mathrm{ml}$ ethanol which restricts the growth of mucoraceous fungi without affecting the other species.

2- Dichloran rose bengal chloramphenicol agar (DRBC), (King et al. 1979): (g/l) peptone 5.0, $\mathrm{KH}_{2} \mathrm{PO}_{4} 1.0, \mathrm{Mg} \mathrm{SO} \mathrm{SO}_{4}$ 0.5, glucose 10.0, agar 15.0, to which rose bengal $(25 \mu \mathrm{g} / \mathrm{ml})$ and chloramphenicol $(100 \mu \mathrm{g} / \mathrm{ml})$ as bacteriostatic agents (Smith \& Dawson 1944, Al- Doory 1980) and dichloran $(20 \mu \mathrm{g} / \mathrm{ml})$ were used.

\section{Identification of filamentous fungi}

The following references were used for the identification of fungal genera and species (purely morphologically, based on macroscopic and microscopic features): Booth (1971), Ellis (1971, 1976), Pitt (1979); Domsch et al. (2007); Moubasher (1993), de Hoog et al. (2000), Schroers (2001), Samson \& Frisvad (2004), Zare \& Gams (2004), Leslie \& Summerell (2006), Crous et al. (2007), Samson \& Varga (2007), Simmons (2007), and De Seifert et al. (2011).

\section{Identification of yeasts}

\section{Morphological characters}

Formation of pseudomycelium and true mycelium (Wickerham 1951) and the ability to form ascospores on three sporulation media (corn meal agar, potato glucose agar and yeast extract malt extract agar at $25^{\circ} \mathrm{C}$ ) (Barnett et al. 2000) were tested.

\section{Physiological characters}

Fermentation of 14 different sugars (D-glucose, D-galactose, maltose, Me- $\alpha$-D-glucoside, sucrose, trehalose, melibiose, lactose, cellobiose, melezitose, raffinose, inulin, starch and D-xylose) and oxidative utilization of 36 carbon compounds were performed according to Barnett et al. (2000). Assimilation of 9 nitrogen compounds (potassium nitrate, sodium nitrite, ethylamine- $\mathrm{HCl}$, L-lysine-HCl, creatine, creatinine, D-glucosamine, imidazole, or D-tryptophan) was determined (Suh et al. 2008).

Hydrolysis of urea, growth at high osmotic pressure, growth at different temperatures, growth in the presence of cycloheximide, diazonium blue B (DBB) test and production of extracellular starch-like compounds were tested.

Identification keys of Barnett et al. (2000) were followed to assign each isolate to its species level. Confirmations of these identifications were carried out using the molecular technique.

\section{Molecular methods}

\section{Growth of the fungus and DNA extraction}

The fungus was grown on Czapek yeast extract agar (CYA) plates and incubated at $25^{\circ} \mathrm{C}$ for 7 days (for filamentous fungal isolates) and on yeast extract malt extract agar (YMA) plates and incubated at $25^{\circ} \mathrm{C}$ for 2 days (for yeast isolates). A small amount of fungal growth was scraped and suspended in $100 \mu \mathrm{l}$ of distilled water and boiled at $100^{\circ} \mathrm{C}$ for 15 minutes and stored at $-70^{\circ} \mathrm{C}$, and sent to SolGent Company, South Korea, for PCR and rDNA sequencing. 
Fungal DNA was extracted and isolated using SolGent purification bead in SolGent Company (Daejeon, South Korea). Internal transcribed spacer (ITS) sequences of nuclear ribosomal DNA were amplified using primers ITS1, ITS4 as follow: universal primer ITS 1 (5' TCC GTA GGT GAA CCT GCG G - 3'), and ITS 4 (5'- TCC TCC GCT TAT TGA TAT GC -3'). Then ampilification was performed using the polymerase chain reaction (PCR) (ABI, 9700). The PCR reaction mixtures were prepared using Solgent EF-Taq as follows: 10X EF-Taq buffer $2.5 \mu 1$, $10 \mathrm{mM}$ dNTPs (T) $0.5 \mu \mathrm{l}$, primer (F-10p) $1.0 \mu \mathrm{l}$, primer (R-10p) $1.0 \mu \mathrm{l}$, EF-Taq (2.5U) $0.25 \mu 1$, template $1.0 \mu \mathrm{l}$, distilled water to $25 \mu \mathrm{l}$. Then the amplification was carried out using the following PCR reaction conditions: one round of amplification consisting of denaturation at $95^{\circ} \mathrm{C}$ for 15 min followed by 30 cycles of denaturation at $95{ }^{\circ} \mathrm{C}$ for $20 \mathrm{sec}$, annealing at $50{ }^{\circ} \mathrm{C}$ for $40 \mathrm{sec}$ and extension at $72{ }^{\circ} \mathrm{C}$ for $1 \mathrm{~min}$, with a final extension step of $72{ }^{\circ} \mathrm{C}$ for $5 \mathrm{~min}$.

The PCR products were then purified with the SolGent PCR Purification Kit-Ultra (SolGent, Daejeon, South Korea) prior to sequencing. Then the purified PCR products were reconfirmed (using size marker) by electrophoreses of the PCR products on $1 \%$ agarose gel. Then these bands were eluted and sequenced. Each sample was sequenced in the sense and antisense direction.

\section{Phylogenetic analysis}

Contigs were created from the sequence data using CLCBio Main Workbench program. The sequence obtained from each isolate was further analyzed using BLAST from the National Center of Biotechnology Information (NCBI) website. Sequences obtained with those retrieved from GenBank database were subjected to Clustal W analysis using MegAlign (DNAStar) software version 5.05 for the phylogenetic analysis. Sequence data were deposited in GenBank and accession numbers are given for them.

Statistical analysis - Descriptive statistical analysis was employed (PC-ORD) to analyze mycobiotic data obtained in both habitats (McCune and Mefford 1999).

\section{Results}

Identification of fungal genera and species was performed using the morphological and microscopical characteristics in addition to the biochemical in case of yeasts (Table 1). In suspected isolates, molecular techniques [Internal transcribed spacer (ITS) sequences of nuclear ribosomal DNA were amplified using primers ITS1, ITS4] were employed that either confirm the previous methods or disagree with them, and in the latter cases they are registered as unidentified (Table 2).

From the biochemical and physiological data presented in Table (1), it is obvious that only the two ascomycetous yeast species (Candida parapsilosis and Meyerozyma guilliermondii) could ferment D-glucose. None of basidiomycetous species could ferment any of the 14 sugars tested. In addition, of the ascomycetous species $M$. guilliermondii could also ferment sucrose and raffinose. On the other hand, the 10 basidiomycetous species tested could be differentiated from the ascomycetous species by their ability to hydrolyze urea and to give positive diazonium blue B reaction. Some other assimilation tests of carbon and nitrogen compounds could differentiate between species (Table 1).

A total of 176 and 139 species and 2 varieties belonging to 66 and 58 genera were recovered, respectively from the air of citrus and grapevine plantations ( 3 farms in the six trips) bimonthly during the period from April 2008 to February 2009. From these, 139 and 108 species and 2 varieties belonging to 59 and 46 genera were recorded on yeast extract malt extract agar supplemented with dichloran (DYM), and 135 and 89 species +2 or 1 variety related to 58 and 44 genera on dichloran rose bengal chloramphenicol agar (DRBC) from the air of the two plants. The filamentous fungi constituted the greatest part of propagules $(96.08 \%$ and $94.03 \%$ on DYM and $98.67 \%$ and $98.49 \%$ on DRBC) while yeasts contributed $3.92 \%$ and $5.97 \%$ and $1.33 \%$ and $1.51 \%$ of total fungi respectively (Table 3). 
Table 1 Physiological comparison of the strains tested of the recorded species.

Species: 1 Candida parapsilosis AUMC7750, 2 Meyerozyma guilliermondii (=Pichia guilliermondii) (anamorph:Candida guilliermondii) AUMC7771, 3 Cryptococcus flavescens AUMC7794, 4 C. magnus AUMC 7793, 5 Melanopsichium pennsylvanicum AUMC7785, 6 Pseudozyma hubiensis AUMC7786, 7 Rhodosporidium paludigenum (anamorph: Rhodotorula graminis) AUMC7783, 8 Rhodotorula glutinis AUMC 7774, 9 R. glutinis AUMC 7776, 10 Rhodotorula mucilaginosa AUMC7778, 11 R. mucilaginosa AUMC7782, 12 Sporidiobolus metaroseus (anamorph: Sporobolomyces roseus) AUMC7788, 13 Sporidiobolus ruineniae (anamorph: Sporobolomyces coprophilous) AUMC7773, 14 S. ruineniae AUMC7781, 15 Trichosporon asahii AUMC7779.

\begin{tabular}{|c|c|c|c|c|c|c|c|c|c|c|c|c|c|c|c|c|}
\hline Species no. & $\mathbf{S}$ & 1 & 2 & 3 & 4 & 5 & 6 & 7 & 8 & 9 & 10 & 11 & 12 & 13 & 14 & 15 \\
\hline \multicolumn{17}{|l|}{ Fermentation } \\
\hline D- glucose & $\mathrm{F} 1$ & $\mathrm{~d}$ & $\mathrm{~d}$ & - & - & - & - & - & - & - & - & - & - & - & - & - \\
\hline Sucrose & F5 & - & $\mathrm{d}$ & - & - & - & - & - & - & - & - & - & - & - & - & - \\
\hline Raffinose & F11 & - & $\mathrm{d}$ & - & - & - & - & - & - & - & - & - & - & - & - & - \\
\hline \multicolumn{17}{|l|}{ Assimilation } \\
\hline D-glucose & $\mathrm{C} 1$ & + & + & + & + & + & + & + & + & + & + & + & + & + & + & + \\
\hline D-galactose & $\mathrm{C} 2$ & + & + & + & + & + & + & $\mathrm{d}$ & $\mathrm{d}$ & $\mathrm{d}$ & + & + & $\mathrm{w}$ & d & + & + \\
\hline L-sorbose & $\mathrm{C} 3$ & + & + & - & $\mathrm{d}$ & $\mathrm{d}$ & $\mathrm{w}$ & $\mathrm{d}$ & $\mathrm{w}$ & $\mathrm{d}$ & - & - & + & + & + & $\mathrm{d}$ \\
\hline D-ribose & $\mathrm{C} 5$ & - & + & $\mathrm{d}$ & $\mathrm{d}$ & + & d & + & $\mathrm{d}$ & + & + & + & + & + & + & + \\
\hline D-xylose & C6 & + & + & + & + & + & + & + & + & + & d & + & $\mathrm{d}$ & + & + & + \\
\hline L-arabinose & $\mathrm{C} 7$ & + & + & + & + & + & + & & & & & & & & & + \\
\hline L-rhamnose & C9 & - & + & + & + & + & - & $\mathrm{d}$ & - & - & + & + & - & d & + & + \\
\hline Sucrose & $\mathrm{C} 10$ & + & + & + & + & + & + & + & + & + & + & + & + & + & + & + \\
\hline Maltose & $\mathrm{C} 11$ & + & + & + & + & + & + & d & + & $\mathrm{d}$ & + & d & + & d & + & + \\
\hline$\alpha, \alpha$-trehalose & $\mathrm{C} 12$ & + & + & + & + & + & + & + & + & + & + & $+\mathrm{w}$ & $+\mathrm{w}$ & + & + & + \\
\hline Methyl- $\alpha$-D-glucoside & $\mathrm{C} 13$ & + & + & + & + & + & + & $\mathrm{d}$ & $\mathrm{d}$ & - & - & - & $\mathrm{d}$ & $\mathrm{d}$ & - & + \\
\hline Cellobiose & $\mathrm{C} 14$ & - & + & + & + & $\mathrm{d}$ & + & & & & & & & & & + \\
\hline Salicin & $\mathrm{C} 15$ & - & + & & $\mathrm{d}$ & & & & & & & & & & & \\
\hline Arbutin & $\mathrm{C} 16$ & - & + & & $\mathrm{d}$ & & & & & & & & & & & \\
\hline Lactose & $\mathrm{C} 18$ & - & $\mathrm{d}$ & + & + & $\mathrm{d}$ & $\mathrm{d}$ & - & - & - & - & - & - & - & - & + \\
\hline Raffinose & C19 & - & + & + & + & + & + & & & & & & & & & - \\
\hline Melezitose & $\mathrm{C} 20$ & + & + & + & + & $\mathrm{w}$ & + & - & + & + & + & $\mathrm{d}$ & + & - & - & + \\
\hline Inulin & $\mathrm{C} 21$ & - & + & + & + & + & + & + & + & + & + & + & d & d & + & $+\mathrm{w}$ \\
\hline Soluble starch & $\mathrm{C} 22$ & - & + & + & + & + & + & - & + & + & d & - & $\mathrm{d}$ & - & - & + \\
\hline Glycerol & $\mathrm{C} 23$ & + & + & & $\mathrm{d}$ & & & & & & & & & & & \\
\hline Meso-erythritol & $\mathrm{C} 24$ & $\mathrm{~d}$ & $\mathrm{~d}$ & $\mathrm{w}$ & - & $\mathrm{d}$ & + & - & - & - & - & - & + & - & - & + \\
\hline Xylitol & $\mathrm{C} 26$ & d & + & & $\mathrm{d}$ & & & & & & & & & & & \\
\hline D-glucitol & $\mathrm{C} 28$ & + & + & $\mathrm{d}$ & $\mathrm{d}$ & $\mathrm{d}$ & + & + & $\mathrm{d}$ & + & + & $\mathrm{d}$ & $\mathrm{d}$ & + & + & + \\
\hline D-mannitol & $\mathrm{C} 29$ & + & + & + & + & + & + & + & + & + & + & + & - & + & + & $\mathrm{d}$ \\
\hline Galactitol & C30 & - & + & $\mathrm{d}$ & $\mathrm{d}$ & - & - & + & - & - & - & $+\mathrm{w}$ & - & + & + & - \\
\hline Myo-inositol & $\mathrm{C} 31$ & - & $\mathrm{d}$ & + & + & $\mathrm{d}$ & d & - & - & - & - & - & - & - & - & + \\
\hline Glucono-d-lactone & $\mathrm{C} 32$ & + & $\mathrm{d}$ & $\mathrm{d}$ & - & + & $\mathrm{d}$ & + & d & $\mathrm{d}$ & + & + & $\mathrm{d}$ & + & + & + \\
\hline D-glucuronate & $\mathrm{C} 36$ & - & $\mathrm{d}$ & + & + & $\mathrm{d}$ & + & - & - & - & - & - & $\mathrm{d}$ & - & - & + \\
\hline D-galacturonate & C37 & - & - & + & $\mathrm{d}$ & + & $\mathrm{w}$ & - & $\mathrm{w}$ & + & d & $d$ & - & + & $\mathrm{d}$ & - \\
\hline Succinate & C39 & + & + & & $\mathrm{d}$ & & & & & & & & & & & \\
\hline Citrate & $\mathrm{C} 40$ & + & + & + & + & $\mathrm{d}$ & + & $\mathrm{d}$ & + & + & + & $\mathrm{d}$ & $\mathrm{d}$ & + & $\mathrm{d}$ & + \\
\hline Methanol & $\mathrm{C} 41$ & $\mathrm{w}$ & $\mathrm{w}$ & - & - & - & $\mathrm{w}$ & - & - & - & w & - & - & - & - & - \\
\hline Ethanol & $\mathrm{C} 42$ & + & + & + & - & + & + & + & + & $\mathrm{d}$ & + & + & d & + & + & + \\
\hline Propane 1,2 diol & $\mathrm{C} 43$ & - & - & - & - & - & - & - & - & - & - & - & - & - & - & - \\
\hline Butane 2,3 diol & $\mathrm{C} 44$ & - & - & - & - & $\mathrm{d}$ & - & - & - & - & - & - & - & - & - & $\mathrm{d}$ \\
\hline Quinic acid & $\mathrm{C} 45$ & - & - & - & $\mathrm{d}$ & + & + & + & + & + & + & $+\mathrm{w}$ & + & + & + & - \\
\hline \multicolumn{17}{|l|}{ Nitrogen compounds } \\
\hline Nitrate & N1 & - & - & - & + & + & + & + & + & + & - & - & + & + & + & - \\
\hline Nitrite & $\mathrm{N} 2$ & - & - & + & + & + & + & + & + & + & - & - & w & + & + & + \\
\hline Ethylamine & $\mathrm{N} 3$ & + & + & + & - & + & + & + & + & + & + & + & w & + & + & - \\
\hline L-lysine & N4 & + & + & + & - & + & + & - & $+w$ & + & w & - & - & + & + & + \\
\hline Creatine & N6 & - & - & - & - & - & - & - & - & - & - & - & - & - & - & - \\
\hline
\end{tabular}




\begin{tabular}{|c|c|c|c|c|c|c|c|c|c|c|c|c|c|c|c|c|}
\hline Species no. & $\mathbf{S}$ & 1 & 2 & 3 & 4 & 5 & 6 & 7 & 8 & 9 & 10 & 11 & 12 & 13 & 14 & 15 \\
\hline Creatinine & N7 & - & - & - & - & - & - & - & - & - & - & - & - & - & - & - \\
\hline D-glucosamine & N8 & - & - & + & $\mathrm{w}$ & + & + & - & - & - & - & - & - & - & - & - \\
\hline Imidazole & N9 & - & - & - & - & - & - & - & - & - & - & - & - & - & - & - \\
\hline D-tryptophane & N10 & - & - & + & - & - & $\mathrm{d}$ & $\mathrm{w}$ & $\mathrm{d}$ & $\mathrm{d}$ & + & + & - & - & - & + \\
\hline \multicolumn{17}{|l|}{ Miscellaneous } \\
\hline $0.01 \%$ cycloheximide & O1 & - & + & $\mathrm{d}$ & + & + & $\mathrm{d}$ & + & + & + & + & + & + & $\mathrm{d}$ & $\mathrm{d}$ & + \\
\hline $0.1 \%$ cycloheximide & $\mathrm{O} 2$ & - & + & $\mathrm{w}$ & - & + & $\mathrm{d}$ & + & + & + & $\mathrm{d}$ & + & $\mathrm{w}$ & $\mathrm{d}$ & $\mathrm{d}$ & + \\
\hline $50 \%$ D-glucose & $\mathrm{O} 4$ & + & + & - & - & - & - & - & - & - & - & - & - & - & - & - \\
\hline $60 \%$ D-glucose & O5 & + & - & - & - & - & - & - & - & - & - & - & - & - & - & - \\
\hline $10 \% \mathrm{NaCl}$ & O6 & + & + & - & + & - & - & - & - & - & - & - & - & - & - & - \\
\hline $16 \% \mathrm{NaCl}$ & O7 & - & - & - & + & - & - & - & - & - & - & - & - & - & - & - \\
\hline Starch forn & M1 & - & - & + & - & 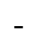 & + & - & - & - & - & - & - & - & - & + \\
\hline Urea hydrolysis & M3 & - & - & + & + & + & + & + & + & + & + & + & + & + & + & + \\
\hline Diazonium blue $\mathrm{B}$ & M4 & - & - & + & + & + & + & + & + & + & + & + & + & + & + & + \\
\hline
\end{tabular}

+: growth; w: weak growth; d, delayed, -: no growth, gap: not tested.

Fermentation of 11 sugars gave negative results for all species tested and were omitted from the table.

From the fungi recovered on DYM, 123 and 96 species and 2 varieties belong to 47 and 37 genera of filamentous fungi and 16 and 14 species related to 12 and 9 genera of yeasts were isolated from the air of both plants, respectively. A total of 10555 and 9505 colony forming units (CFUs) were recovered from the air of citrus and grapevine plantations in 18 exposures (Table 2). The highest CFUs were recorded in the three farms collectively in February and December, while the lowest were registered in June on the two plantations (Figs. 1-4). The broadest spectrum of species in the three farms collectively was observed in June but the narrowest was noticed in February.

From the fungi recovered on DRBC, 120 and 83 species and 2 or 1 variety belong to 45 and 38 genera of filamentous fungi, and 16 and 8 species related to 12 and 6 genera of yeasts were isolated from the air of both plants respectively. A total of 9343 and 4644 CFUs were recovered (Table 2). The highest number of CFUs was recorded in the three farms collectively in February and August, while the lowest in October (Figs. 2 \& 4). The broadest spectrum of species was observed in June and April in the three farms collectively (69 and 42 species) but the narrowest (14 and 9) in October.

Many genera of filamentous fungi were recorded in high frequency of occurrence but varied in their percentage counts from high $(57.57 \%$ and $38.24 \%$, and $53.41 \%$ and $26.29 \%$ on both media for citrus and grapevine plantations respectively) to low (0.24\% and $0.26 \%$, and $0.32 \%$ and $0.24 \%$ ) and these were Cladosporium, Penicillium, Aspergillus, Fusarium, Alternaria, Emericella, Myrothecium, Cochliobolus, Phoma, Pleospora, Setosphaeria, Botryodiplodia, Humicola, Petromyces and Rhizopus (Table 3, Figs 1-4).

Cladosporium was the most common genus in the air of both plants, yielding considerably higher numbers of propagules in citrus plantations $(53.41 \%-57.57 \%$ of total fungi) than those in grapevine plantations $(26.29 \%$ - 38.24\%). Its count peak was regularly recorded in February in the air of citrus plantations on both media but irregularly in the air of grapevine plantations, in December and April on DYM and DRBC respectively. Its trough was registered in August and October on DYM and DRBC respectively in citrus air and in October on both media in grapevine air. In the air of citrus plantations, $C$. cladosporioides was the most common species, contributing about half of total fungi $(46.78 \%-53.80 \%)$. In the air of grapevine it yielded markedly less percentage counts $(23.58 \%-35.09 \%)$. C. sphaerospermum was recorded in high frequency in citrus air on both media but in grapevine air it was isolated in moderate and high frequencies on DYM and DRBC respectively. C. herbarum was isolated only from the air of grapevine plantation, in low frequency. 
Table 2 The Assiut University Mycological Centre accession number (AUMC) of yeast and filamentous fungal [all belonging to Ascomycota except Quambalaria cyanescens to Basidiomycota (Ustilaginomycetes, Quambalariaceae)] strains isolated from air of citrus or grapevine plantations with accession GenBank numbers given together with the closest match in the GenBank database and sequence similarity in percent to the match as inferred from Blastn searches of ITS sequences.

\begin{tabular}{|c|c|c|c|c|c|c|c|}
\hline $\begin{array}{l}\text { AUMC } \\
\text { number }\end{array}$ & $\begin{array}{l}\text { Isolation } \\
\text { source }\end{array}$ & $\begin{array}{l}\text { Accession } \\
\text { GenBank } \\
\text { number }\end{array}$ & $\begin{array}{l}\text { Length } \\
\text { (bp) }\end{array}$ & Closest Genbank match \# ITS & $\begin{array}{l}\text { Sequencing } \\
\text { similarity }(\%)\end{array}$ & Species & References \\
\hline \multicolumn{8}{|c|}{ Filamentous fungi } \\
\hline 5731 & $\begin{array}{l}\text { Grapevine } \\
\text { air }\end{array}$ & JQ425375 & 585 & $\begin{array}{l}\text { EF634410=NRRL 35386T } \\
\text { GU092949=NRRL 58014T }\end{array}$ & $\begin{array}{l}100 \\
100\end{array}$ & $\begin{array}{l}\text { Hamigera insecticola } \\
\text { Hamigera inflata }\end{array}$ & Peterson et al. 2010 \\
\hline 6293 & Citrus air & JQ425376 & 645 & DQ317622= CBS357.73 & 100 & Quambalaria cyanescens & de Beer et al. 2006 \\
\hline 6707 & Citrus air & JQ425377 & 556 & HQ897807=DAOM:195309 & 93 & Pseudonectria pachysandricola & Gräfenhan et al. 2011 \\
\hline 5748 & $\begin{array}{l}\text { Grapevine } \\
\text { air }\end{array}$ & JN393254 & 565 & $\begin{array}{l}\text { GQ352493=5GP } / \mathrm{T} \\
\text { EU833205=A1.9 } \\
\text { FJ416300 }=1002 \mathrm{~F} 2 \\
\text { GU595031 }=\mathrm{H} 4307\end{array}$ & 99 & $\begin{array}{l}\text { Aspergillus sp. } \\
\text { Aspergillus aculeatus } \\
\text { Aspergillus sp. } \\
\text { Aspergillus } \mathrm{sp} .\end{array}$ & $\begin{array}{l}\text { Moubasher \& Zeinab } \\
\text { Soliman } 2011 \text { (as Aspergillus } \\
\text { assiutensis) }\end{array}$ \\
\hline 5716 & Citrus air & JQ425381 & 558 & GQ169452 = N11 & 100 & Pleosporaceae sp. & \\
\hline 6241 & Citrus air & JQ425386 & 596 & $\begin{array}{l}\text { HQ909075= } \\
\text { GU291796=CBS125525 } \\
\text { AY924288 = AZ-32 }\end{array}$ & $\begin{array}{l}100 \\
99\end{array}$ & $\begin{array}{l}\text { Bartalinia sp. } \\
\text { Bartalinia pondoensis } \\
\text { Bartalinia robillardoides }\end{array}$ & $\begin{array}{l}\text { Suryanarayanan et al. 2011, } \\
\text { Marincowitz et al. } 2010\end{array}$ \\
\hline \multicolumn{8}{|c|}{ Yeast fungi } \\
\hline $\begin{array}{l}\text { No } 15 \\
\text { (dead) }\end{array}$ & $\begin{array}{l}\text { Grapevine } \\
\text { air }\end{array}$ & JQ425363 & 608 & $\begin{array}{l}\text { AY070006 }=\text { AS 2.2108 } \\
\text { EU003482 }=\text { CBS } 7683^{T}\end{array}$ & $\begin{array}{l}100 \\
99\end{array}$ & $\begin{array}{l}\text { Sporidiobolus metaroseus (anamorph: } \\
\text { Sporobolomyces roseus) }\end{array}$ & Valerio et al. 2008 \\
\hline 7788 & Citrus air & JQ425365 & 582 & $\begin{array}{l}\text { AY015435 }=\text { CBS5541 } \\
\text { EU003482=CBS7683 }\end{array}$ & $\begin{array}{l}90 \\
89\end{array}$ & $\begin{array}{l}\text { Sporidiobolus metaroseus (anam: } \\
\text { Sporobolomyces roseus) }\end{array}$ & Valerio et al. 2008 \\
\hline 7785 & Citrus air & JQ425368 & 777 & AY740040 & 96 & Melanopsichium pennsylvanicum & Stoll et al. 2005 \\
\hline 7793 & $\begin{array}{l}\text { Grapevine } \\
\text { air }\end{array}$ & JQ425369 & 612 & $\mathrm{AF} 190008=\mathrm{CBS} 140^{\mathrm{T}}$ & 89 & Cryptococcus magnus & Fell et al. 2000 \\
\hline 7776 & Citrus air & JQ425370 & 618 & HQ670677 & 99 & Rhodotorula glutinis & Yang et al. 2011 \\
\hline 7778 & Citrus air & JQ425392 & 629 & $\begin{array}{l}\text { HQ909092 }=\text { KDLYC24-1 } \\
\text { AF444541 }=\text { CBS } 316^{\mathrm{T}}\end{array}$ & 99 & Rhodotorula mucilaginosa & Scorzetti et al. 2002 \\
\hline 7781 & Citrus air & JQ425394 & 610 & AY015433 = CBS 5001 ${ }^{\mathrm{T}}$ & 99 & $\begin{array}{l}\text { Sporidiobolus ruineniae (anamorph: } \\
\text { Sporobolomyces coprophilous) }\end{array}$ & Fell et al. 2002 \\
\hline 7783 & Citrus air & JQ425395 & 616 & $\begin{array}{l}\text { AF444493 }=\text { CBS } 6567 \\
\text { AF444492 }=\text { CBS } 6566^{\mathrm{T}}\end{array}$ & $\begin{array}{l}99 \\
99\end{array}$ & $\begin{array}{l}\text { Rhodosporidium paludigenum } \\
\text { (anamorph: Rhodotorula graminis) }\end{array}$ & Scorzetti et al. 2002 \\
\hline 7774 & $\begin{array}{l}\text { Grapevine } \\
\text { air }\end{array}$ & JQ425397 & 618 & $\begin{array}{l}\text { HQ670677 } \\
\text { EF194846 =MCCC2E00215 }\end{array}$ & 99 & Rhodotorula glutinis & Yang et al. 2011 \\
\hline
\end{tabular}




\begin{tabular}{|c|c|c|c|c|c|c|c|}
\hline $\begin{array}{l}\text { AUMC } \\
\text { number }\end{array}$ & $\begin{array}{l}\text { Isolation } \\
\text { source }\end{array}$ & $\begin{array}{c}\text { Accession } \\
\text { GenBank } \\
\text { number }\end{array}$ & $\begin{array}{l}\text { Length } \\
\text { (bp) }\end{array}$ & Closest Genbank match \# ITS & $\begin{array}{l}\text { Sequencing } \\
\text { similarity }(\%)\end{array}$ & Species & References \\
\hline 7782 & $\begin{array}{l}\text { Grapevine } \\
\text { air }\end{array}$ & JQ425399 & 633 & $\begin{array}{l}\text { HQ909092 =_KDLYC24-1 } \\
\text { AF444541 }=\text { CBS 316 }\end{array}$ & $\begin{array}{l}99 \\
98\end{array}$ & Rhodotorula mucilaginosa & Scorzetti et al. 2002 \\
\hline 7794 & $\begin{array}{l}\text { Grapevine } \\
\text { air }\end{array}$ & JQ425400 & 539 & $\begin{array}{l}\text { FN428902 = IMUFRJ } 51986 \\
\text { AM176643 }\end{array}$ & 99 & Cryptococcus flavescens & Molnár \& Prillinger 2005 \\
\hline 7779 & $\begin{array}{l}\text { Grapevine } \\
\text { air }\end{array}$ & JQ425402 & 553 & $\begin{array}{l}\text { AM900369 = YS124 } \\
\text { FJ943429 = CBS } 2479^{\mathrm{T}}\end{array}$ & 99 & Trichosporon asahii & \\
\hline 7773 & Citrus air & JQ425373 & 613 & $\mathrm{AY} 015433=\mathrm{CBS} 5001^{\mathrm{T}}$ & 99 & $\begin{array}{l}\text { Sporidiobolus ruineniae (anamorph: } \\
\text { Sporobolomyces coprophilous) }\end{array}$ & Fell et al. 2002 \\
\hline 7786 & Citrus air & JQ425374 & 987 & $\begin{array}{l}\text { DQ008954=CBS } 10077^{\mathrm{T}} \\
\text { HQ832814 = LH146 }\end{array}$ & $\begin{array}{l}98 \\
97\end{array}$ & Pseudozyma hubeiensis & Wang et. al. 2006 \\
\hline 7263 & Citrus air & JQ425353 & 620 & $\begin{array}{l}\text { EF197943 }=\text { HK67-4 } \\
\text { EF190231 }=\text { wwl-2 } 1\end{array}$ & 100 & $\begin{array}{l}\text { Debaryomyces hansenii } \\
\text { (anamorph:Candida famata) }\end{array}$ & \\
\hline 7750 & Citrus air & JQ425354 & 503 & FJ872016 = CBS 604 ${ }^{\underline{T}}$ & 100 & Candida parapsilosis & \\
\hline 7771 & $\begin{array}{l}\text { Grapevine } \\
\text { air }\end{array}$ & JQ425356 & 590 & $\begin{array}{l}\text { EF197814 = HK53 } \\
\text { EU568971=CNRMA200500864 }\end{array}$ & 100 & $\begin{array}{l}\text { Meyerozyma guilliermondii (=Pichia } \\
\text { guilliermondii) (anamorph:Candida } \\
\text { guilliermondii) }\end{array}$ & Desnos-Ollivier et al. 2008 \\
\hline 7264 & Citrus air & JQ425359 & 635 & $\begin{array}{l}\text { EF197943= HK67-4 } \\
\text { AB220029 = IFM 54258 }\end{array}$ & 100 & $\begin{array}{l}\text { Debaryomyces hansenii } \\
\text { D. nepalensis }\end{array}$ & Moretti et al. 2007 \\
\hline
\end{tabular}

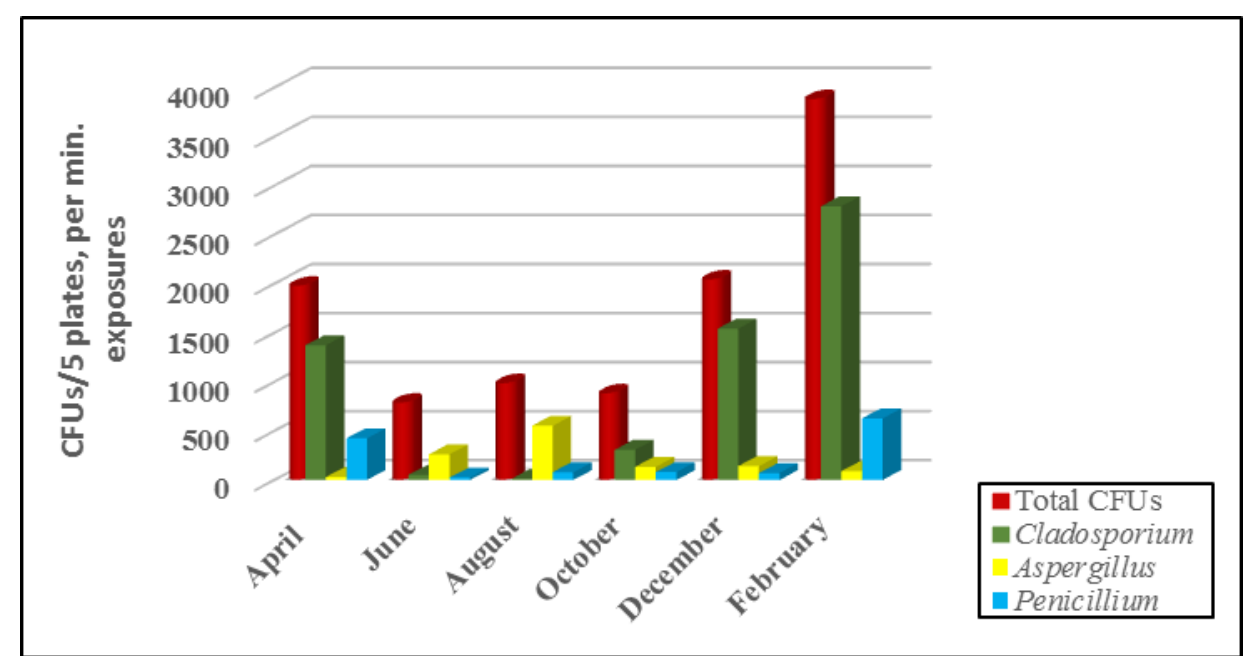

Fig. 1 - Bimonthly counts of common fungi in the air of citrus plantations on DYM during the period of study. 
Aspergillus was one of the most common genera in both plants contributing relatively higher numbers of propagules in the air of grapevine plantations (25.26\% $-25.77 \%$ of total fungi) than those from citrus plantations (11.47\% - $13.98 \%)$. Its peak was regularly recorded in August in both plants on both media while its trough was registered in April and October on DYM and DRBC respectively in citrus air and in December on both media in grapevine. Fifteen species of Aspergillus were recorded from citrus air while two species were isolated from grapevine only. Species of Aspergillus belonging to section Nigri (A. aculeatinus, A. aculeatus, A. brasiliensis and A. niger) were the most common among its species in the air of grapevine plantations contributing relatively large proportions of Aspergillus count compared with those found in citrus air. On the other hand, A. ochraceus was the most common species in the air of citrus, contributing large numbers of propagules while it was recorded in moderate and low frequencies in grapevine air.

Alternaria was the second most frequent genus behind Aspergillus in grapevine air and after Aspergillus and Cladosporium in citrus air, yielding considerably higher numbers of propagules in the air of grapevine plantations (13.41\%-17.44\% of total fungi) than those from citrus plantations $(2.69 \%-2.70 \%)$. Its peak was recorded in February and December in the air of citrus plantations on DYM and on DRBC respectively but regularly in December in the air of grapevine plantations on both media. Its trough was registered in August on both media in citrus air and in April and June on DYM and DRBC respectively in grapevine. In the air of both plantations, A. alternata was the most common, contributing considerably higher percentage counts of total fungi in grapevine air $(10.45 \%-13.65 \%)$ than those of citrus air $(2.23 \%-2.35 \%)$. A. citri was recorded in moderate and low frequencies in citrus air on DYM and DRBC respectively while it was missed in grapevine air. A. astragali, A. nucis, A. raphani, A. tenuissima, and A. tuberculata were isolated only from the air of grapevine plantations.

Fusarium was of high frequency in citrus air, yielding small numbers of propagules $(1.22 \%$ - $2.92 \%$ of total fungi). In grapevine plantations, it was isolated in high or moderate frequency, contributing $1.67 \%-0.84 \%$ of total fungi. Its peak was regularly recorded in June in the air of citrus plantations on both media but irregularly in the air of grapevine plantations, in October and August on DYM and DRBC respectively. Its trough was registered in October and April in citrus air on DYM and DRBC respectively and in June and February in grapevine air. In the air of citrus plantations, 9 species were identified of which $F$. semitectum was the most common species, contributing $0.56 \%-1.90 \%$ of total fungi. In the air of grapevine it was recorded in high or moderate frequency, yielding $0.43 \%-1.22 \%$ of total fungi. F. equiseti and $F$. concolor were recorded in high or moderate frequency in citrus air, while in grapevine air, $F$. equiseti was isolated in moderate frequency and $F$. concolor in rare frequency on DYM only. F. camptoceras and $F$. torulosum were isolated from citrus air only while $F$. chlamydosporum, $F$. circinatum, $F$. konzum, $F$. proliferatum, $F$. pseudonygami and F.scripi were isolated from grapevine air only.

Penicillium was one of the most common genera contributing noticeably higher numbers of propagules in the air of citrus plantations $(12.38 \%$ and $19.99 \%$ of total fungi on DYM and DRBC respectively) than those from grapevine plantations $(3.34 \%-5.51 \%)$. Its peak was recorded in February and April on DYM and DRBC respectively in citrus air and in August on both media in grapevine air, while its trough was registered in June and October on DYM and DRBC respectively in citrus air and in December on both media in grapevine. In citrus air, Penicillium olsonii was the most common species contributing $7.54 \%-10.24 \%$ of total fungi while it was missed in grapevine air. P. citrinum was isolated in high or moderate frequency from citrus air but was recorded in rare frequency on both media from grapevine air. On the other hand, P. oxalicum was the most common species in the air of grapevine, contributing relatively the largest proportions of Penicillium counts (3.03\% $-5.28 \%$ of total fungi) while it was recorded in low frequency in citrus air. Fifteen species of Penicillium were recorded in citrus air while two species in grapevine only (Table 2).

Cochliobolus ( 2 species) was recorded in high frequency in the air of both plantations. The most prevalent species was $C$. lunatus followed by $C$. australiensis. Curvularia (4 species) was isolated in low frequency from the air of both plantations. 


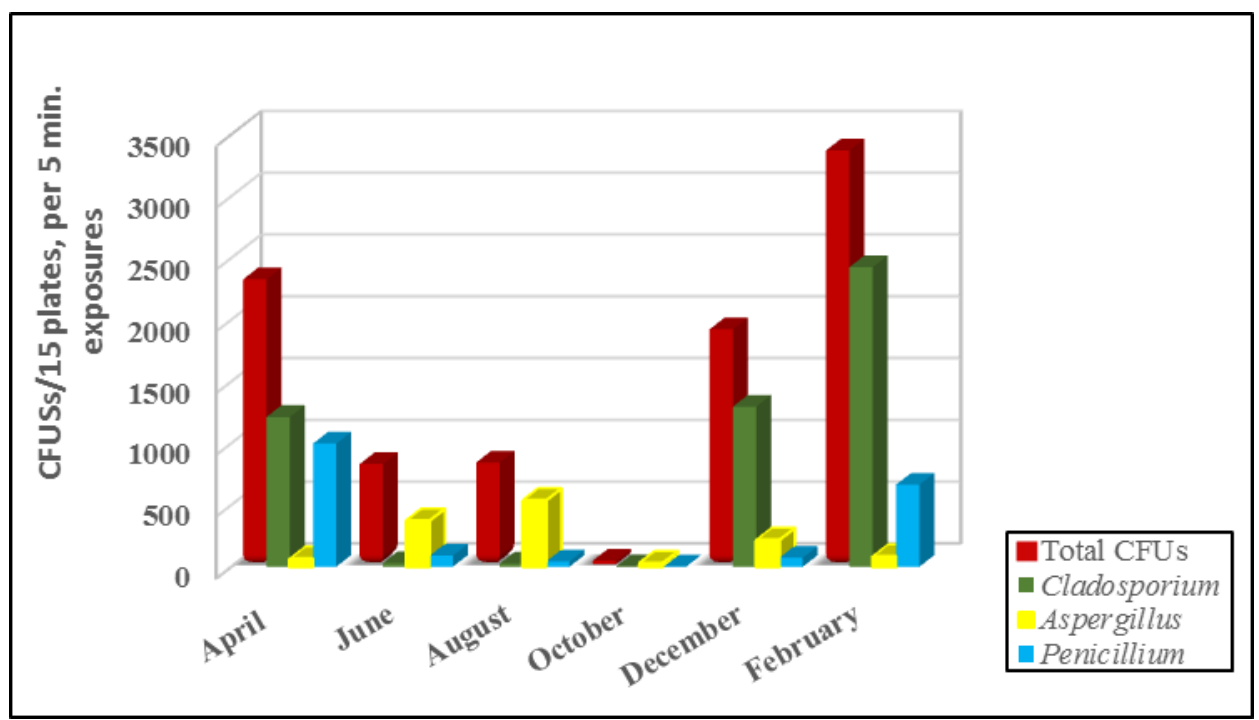

Fig. 2 - Bimonthly counts of common fungi in the air of citrus plantations on DRBC during the period of study.

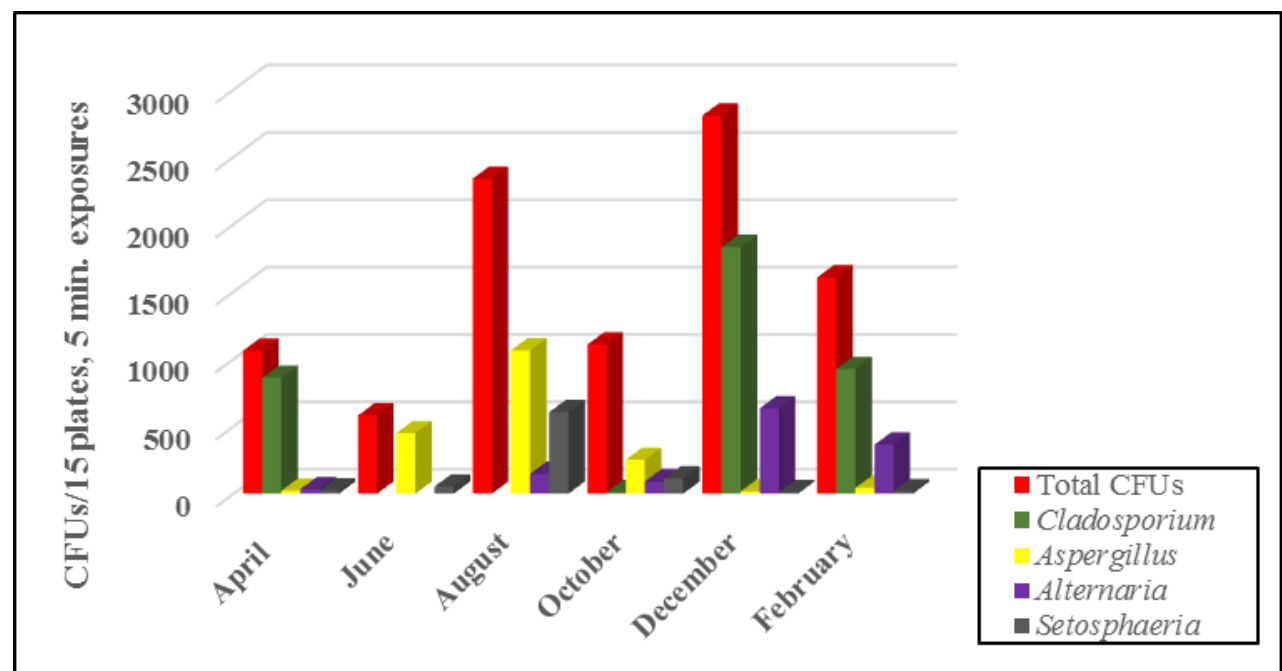

Fig. 3 - Bimonthly counts of common fungi in the air of grapevine plantations on DYM during the period of study.

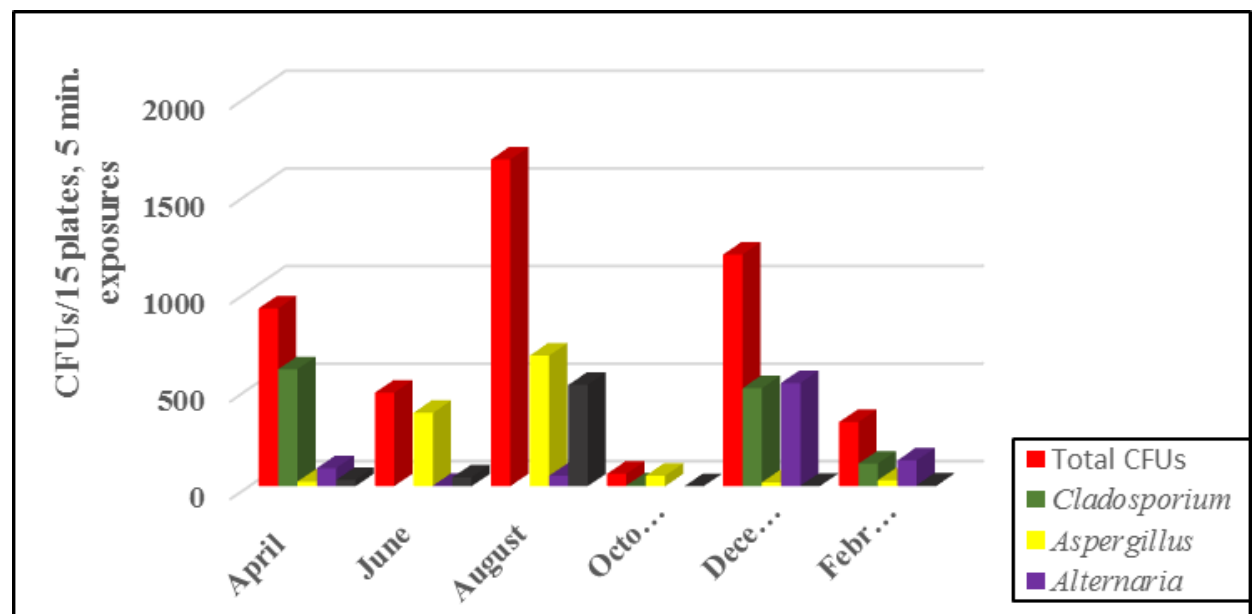

Fig. 4 - Bimonthly counts of common fungi in the air of grapevine plantations on DRBC during the period of study. 
Table 3 Collective data of counts (CFU), percentage counts (CFU\%) calculated to total fungi, frequency (F) and occurrence remarks (O) of fungi recovered from the air of citrus and grapevine plantations on DYM and DRBC agar media bimonthly, during the period from April 2008February 2009 (counts of CFU calculated per 5 minutes exposures in each sample, collectively in 18 samples in each plantation).

\begin{tabular}{|c|c|c|c|c|c|c|c|c|c|c|c|c|}
\hline & \multicolumn{5}{|c|}{ Citrus } & \multicolumn{7}{|c|}{ Grapevine } \\
\hline & \multicolumn{2}{|c|}{ DYM } & \multicolumn{4}{|c|}{ DRBC } & \multicolumn{2}{|c|}{ DYM } & \multicolumn{4}{|c|}{ DRBC } \\
\hline & $\mathrm{CFU}$ & $\% \mathrm{CFU}$ & $\mathrm{F} \& \mathrm{O}$ & $\mathrm{CFU}$ & $\% \mathrm{CFU}$ & $\mathrm{F} \& \mathrm{O}$ & CFU & $\% \mathrm{CFU}$ & $\mathrm{F} \& \mathrm{O}$ & CFU & $\% \mathrm{CFU}$ & $\mathrm{F} \& \mathrm{O}$ \\
\hline Filamentous fungi & 10140 & 96.08 & $18 \mathrm{H}$ & 9219 & 98.67 & $18 \mathrm{H}$ & 8947 & 94.03 & $18 \mathrm{H}$ & 4574 & 98.49 & $18 \mathrm{H}$ \\
\hline Absidia & 15 & 0.14 & $6 \mathrm{M}$ & 6 & 0.06 & $3 \mathrm{~L}$ & & & & 1 & 0.02 & $1 \mathrm{R}$ \\
\hline A. cylindrospora & 14 & 0.13 & $5 \mathrm{M}$ & 6 & 0.06 & $3 \mathrm{~L}$ & & & & 1 & 0.02 & $1 \mathrm{R}$ \\
\hline Acremonium & 1 & 0.01 & $1 \mathrm{R}$ & 2 & 0.02 & $2 \mathrm{R}$ & 11 & 0.12 & $4 \mathrm{~L}$ & 2 & 0.04 & $1 \mathrm{R}$ \\
\hline A. fusidioides & & & & 1 & 0.01 & $1 \mathrm{R}$ & & & & 2 & 0.04 & $1 \mathrm{R}$ \\
\hline A. hyalinulum & & & & 1 & 0.01 & $1 \mathrm{R}$ & 9 & 0.09 & $3 \mathrm{~L}$ & & & \\
\hline Alternaria & 285 & 2.70 & $18 \mathrm{H}$ & 251 & 2.69 & $11 \mathrm{H}$ & 1274 & 13.41 & $14 \mathrm{H}$ & 810 & 17.44 & $12 \mathrm{H}$ \\
\hline A. alternata & 235 & 2.23 & $18 \mathrm{H}$ & 219 & 2.35 & $11 \mathrm{H}$ & 993 & 10.45 & $13 \mathrm{H}$ & 634 & 13.65 & $11 \mathrm{H}$ \\
\hline A. citri & 28 & 0.27 & $5 \mathrm{M}$ & 4 & 0.04 & $3 \mathrm{~L}$ & & & & & & \\
\hline A. chlamydospora & 8 & 0.08 & $5 \mathrm{M}$ & 5 & 0.05 & $2 \mathrm{R}$ & 88 & 0.91 & $7 \mathrm{M}$ & 114 & 2.45 & $8 \mathrm{M}$ \\
\hline Alternaria spp. & 14 & 0.13 & $4 \mathrm{~L}$ & 23 & 0.24 & $4 \mathrm{~L}$ & 157 & 1.64 & $11 \mathrm{H}$ & 55 & 1.18 & $8 \mathrm{M}$ \\
\hline Aspergillus & 1212 & 11.47 & $18 \mathrm{H}$ & 1306 & 13.98 & $18 \mathrm{H}$ & 1851 & 19.48 & $18 \mathrm{H}$ & 1173 & 25.26 & $17 \mathrm{H}$ \\
\hline A.aculeatinus & & & & & & & 181 & 0.91 & $7 \mathrm{M}$ & 125 & 2.69 & $5 \mathrm{M}$ \\
\hline A. aculeatus & 44 & 0.42 & $6 \mathrm{M}$ & 25 & 0.27 & $4 \mathrm{~L}$ & 322 & 9.70 & $14 \mathrm{H}$ & 183 & 3.94 & $14 \mathrm{H}$ \\
\hline A. auricomus & 8 & 0.08 & $1 \mathrm{R}$ & 1 & 0.01 & $1 \mathrm{R}$ & 3 & 0.03 & $2 \mathrm{R}$ & & & \\
\hline A. brasiliensis & 29 & 0.27 & $10 \mathrm{H}$ & 93 & 0.99 & $9 \mathrm{H}$ & 117 & 1.23 & $9 \mathrm{M}$ & 152 & 3.27 & $10 \mathrm{H}$ \\
\hline A. bridgeri & 7 & 0.07 & $1 \mathrm{R}$ & 3 & 0.03 & $1 \mathrm{R}$ & & & & & & \\
\hline A. calidoustus & 8 & 0.08 & $2 \mathrm{R}$ & 9 & 0.10 & $2 \mathrm{R}$ & & & & 1 & 0.02 & $1 \mathrm{R}$ \\
\hline A. campestris & 14 & 0.13 & $1 \mathrm{R}$ & 4 & 0.04 & $1 \mathrm{R}$ & & & & & & \\
\hline A. candidus & 3 & 0.03 & $1 \mathrm{R}$ & 6 & 0.06 & $2 \mathrm{R}$ & & & & & & \\
\hline A. clavatus & 6 & 0.06 & $4 \mathrm{~L}$ & 2 & 0.02 & $2 \mathrm{R}$ & & & & & & \\
\hline A. dimorphicus & 59 & 0.56 & $6 \mathrm{M}$ & 120 & 1.28 & $7 \mathrm{M}$ & 3 & 0.03 & $2 \mathrm{R}$ & 1 & 0.02 & $1 \mathrm{R}$ \\
\hline A. flavus var. columnaris & 3 & 0.03 & $2 \mathrm{R}$ & 1 & 0.01 & $1 \mathrm{R}$ & 1 & 0.01 & $1 \mathrm{R}$ & & & \\
\hline A. insulicola & 4 & 0.04 & $3 \mathrm{~L}$ & 5 & 0.05 & $2 \mathrm{R}$ & & & & & & \\
\hline A. japonicus & 10 & 0.10 & $2 \mathrm{R}$ & & & & 17 & 0.18 & $2 \mathrm{R}$ & 175 & 3.77 & 2 \\
\hline A. lacticoffeatus & 1 & 0.01 & $1 \mathrm{R}$ & 16 & 0.17 & $3 \mathrm{~L}$ & & & & & & \\
\hline A. niger & 186 & 1.76 & $17 \mathrm{H}$ & 147 & 1.57 & $13 \mathrm{H}$ & 1065 & 11.21 & $18 \mathrm{H}$ & 467 & 10.66 & $16 \mathrm{H}$ \\
\hline A. ochraceus & 729 & 6.91 & $17 \mathrm{H}$ & 700 & 7.49 & $14 \mathrm{H}$ & 45 & 0.47 & $7 \mathrm{M}$ & 13 & 0.28 & $4 \mathrm{~L}$ \\
\hline A. ostianus & 18 & 0.17 & $4 \mathrm{~L}$ & 93 & 0.99 & $2 \mathrm{R}$ & & & & & & \\
\hline A. proliferans & & & & 7 & 0.07 & $1 \mathrm{R}$ & & & & 14 & 0.30 & $1 \mathrm{R}$ \\
\hline A. robustus & 9 & 0.09 & $4 \mathrm{~L}$ & 24 & 0.26 & $4 \mathrm{~L}$ & & & & & & \\
\hline A. sclerotiorum & 5 & 0.05 & $2 \mathrm{R}$ & & & & & & & & & \\
\hline A. sulphureus & 4 & 0.04 & $2 \mathrm{R}$ & & & & & & & & & \\
\hline
\end{tabular}




\begin{tabular}{|c|c|c|c|c|c|c|c|c|c|c|c|c|}
\hline & \multicolumn{5}{|c|}{ Citrus } & \multicolumn{7}{|c|}{ Grapevine } \\
\hline & \multicolumn{2}{|c|}{ DYM } & \multicolumn{4}{|c|}{ DRBC } & \multicolumn{2}{|c|}{ DYM } & \multicolumn{4}{|c|}{ DRBC } \\
\hline & $\mathrm{CFU}$ & $\% \mathrm{CFU}$ & $\mathrm{F} \& \mathrm{O}$ & $\mathrm{CFU}$ & $\% \mathrm{CFU}$ & $\mathrm{F} \& \mathrm{O}$ & $\mathrm{CFU}$ & $\% \mathrm{CFU}$ & $\mathrm{F} \& \mathrm{O}$ & $\mathrm{CFU}$ & $\% \mathrm{CFU}$ & $\mathrm{F} \& \mathrm{O}$ \\
\hline A. sydowii & 5 & 0.05 & $2 \mathrm{R}$ & 1 & 0.01 & $1 \mathrm{R}$ & 5 & 0.05 & $3 \mathrm{R}$ & 2 & 0.04 & $2 \mathrm{R}$ \\
\hline A. terreus & 4 & 0.04 & $3 \mathrm{~L}$ & 7 & 0.07 & $3 \mathrm{~L}$ & 15 & 016 & $5 \mathrm{~L}$ & 7 & 0,15 & $6 \mathrm{M}$ \\
\hline A. terreus var. aureus & 6 & 0.06 & $2 \mathrm{R}$ & 4 & 0.04 & $1 \mathrm{R}$ & 63 & 0.66 & $4 \mathrm{~L}$ & 7 & 0.15 & $1 \mathrm{R}$ \\
\hline A. tubingensis & 37 & 0.35 & $3 \mathrm{~L}$ & 21 & 0.22 & $3 \mathrm{~L}$ & 11 & 0.12 & $1 \mathrm{R}$ & 25 & 0.54 & $2 \mathrm{R}$ \\
\hline A. ustus & 1 & 0.01 & $1 \mathrm{R}$ & 1 & 0.01 & $1 \mathrm{R}$ & 1 & 0.01 & $1 \mathrm{R}$ & & & \\
\hline A. versicolor & 5 & 0.05 & $3 \mathrm{~L}$ & 2 & 0.02 & $2 \mathrm{R}$ & 2 & 0.02 & $1 \mathrm{R}$ & & & \\
\hline Bartalinia pondoensis & 3 & 0.03 & $2 \mathrm{R}$ & & & & & & & & & \\
\hline Basidiomycete sp. & 9 & 0.09 & $3 \mathrm{~L}$ & 3 & 0.03 & $1 \mathrm{R}$ & & & & & & \\
\hline Beltrania querna & 104 & 0.99 & $8 \mathrm{M}$ & 97 & 1.04 & $5 \mathrm{M}$ & & & & & & \\
\hline Bipolaris & & & & 8 & 0.09 & $2 \mathrm{R}$ & 14 & 0.15 & $4 \mathrm{~L}$ & 7 & 0.15 & $2 \mathrm{R}$ \\
\hline B. papendorfii & & & & & & & 10 & 0.11 & $4 \mathrm{~L}$ & 7 & 0.15 & $2 \mathrm{R}$ \\
\hline B. subpapendorfii & & & & 8 & 0.09 & $2 \mathrm{R}$ & 4 & 0.04 & $1 \mathrm{R}$ & & & \\
\hline Botryodiplodia theobromae & 25 & 0.24 & $11 \mathrm{H}$ & 12 & 0.13 & $7 \mathrm{M}$ & 2 & 0.02 & $2 \mathrm{R}$ & 3 & 0.06 & $3 R$ \\
\hline Botryotrichum sp. & 1 & 0.01 & $1 \mathrm{R}$ & 3 & 0.03 & $3 \mathrm{~L}$ & & & & 8 & 0.17 & $2 \mathrm{R}$ \\
\hline Cladosporium & 6076 & 57.57 & $18 \mathrm{H}$ & 4990 & 53.41 & $16 \mathrm{H}$ & 3634 & 38.24 & $11 \mathrm{H}$ & 1221 & 26.29 & $10 \mathrm{H}$ \\
\hline C. cladosporioides & 5678 & 53.80 & $18 \mathrm{H}$ & 4371 & 46.78 & $12 \mathrm{H}$ & 3335 & 35.09 & $11 \mathrm{H}$ & 1095 & 23.53 & $9 \mathrm{H}$ \\
\hline C. oxysporum & 212 & 2.01 & $7 \mathrm{M}$ & 269 & 2.88 & $8 \mathrm{M}$ & 15 & 0.16 & $4 \mathrm{~L}$ & 31 & 0.67 & $6 \mathrm{M}$ \\
\hline C. sphaerospermum & 185 & 1.75 & $12 \mathrm{H}$ & 349 & 3.74 & $14 \mathrm{H}$ & 179 & 1.88 & $6 \mathrm{M}$ & 89 & 1.92 & $9 \mathrm{H}$ \\
\hline C. spongiosum & 1 & 0.01 & $1 \mathrm{R}$ & 1 & 0.01 & $1 \mathrm{R}$ & 105 & 1.11 & $2 \mathrm{R}$ & 2 & 0.04 & $1 \mathrm{R}$ \\
\hline Clonostachys & 6 & 0.06 & $2 \mathrm{R}$ & 3 & 0.03 & $2 \mathrm{R}$ & 3 & 0.03 & $2 \mathrm{R}$ & & & \\
\hline C. rogersoniana & 5 & 0.05 & $1 \mathrm{R}$ & 1 & 0.01 & $1 \mathrm{R}$ & & & & & & \\
\hline C. rosea & & & & & & & 3 & 0.03 & $2 \mathrm{R}$ & & & \\
\hline Cochliobolus & 93 & 0.89 & $10 \mathrm{H}$ & 34 & 0.46 & $5 \mathrm{M}$ & 222 & 2.34 & $13 \mathrm{H}$ & 40 & 0.86 & $9 \mathrm{H}$ \\
\hline C. australiensis & 46 & 0.44 & $6 \mathrm{M}$ & 4 & 0.04 & $2 \mathrm{R}$ & 48 & 0.51 & $6 \mathrm{M}$ & 15 & 0.32 & $5 \mathrm{M}$ \\
\hline C. lunatus & 47 & 0.45 & $10 \mathrm{H}$ & 30 & 0.32 & $4 \mathrm{~L}$ & 172 & 1.81 & $8 \mathrm{M}$ & 23 & 0.50 & $5 \mathrm{M}$ \\
\hline C. tuberculatus & & & & & & & 2 & 0.02 & $1 \mathrm{R}$ & 2 & 0.04 & $2 \mathrm{R}$ \\
\hline Curvularia & 3 & 0.03 & $3 \mathrm{~L}$ & 1 & 0.01 & $1 \mathrm{R}$ & 16 & 0.17 & $3 \mathrm{~L}$ & 1 & 0.02 & $1 \mathrm{R}$ \\
\hline C. clavata & 3 & 0.03 & $3 \mathrm{~L}$ & & & & 15 & 0.16 & $3 \mathrm{~L}$ & 1 & 0.02 & $1 \mathrm{R}$ \\
\hline Cylindrocladium intermedium & 3 & 0.03 & $2 \mathrm{R}$ & & & & & & & & & \\
\hline Dichocladosporium chlorocephalum & 49 & 0.46 & $4 \mathrm{~L}$ & 17 & 0.18 & $5 \mathrm{M}$ & 199 & 2.09 & $5 \mathrm{M}$ & 13 & 0.28 & $4 \mathrm{~L}$ \\
\hline Embellisia didymospora & & & & & & & 6 & 0.06 & $3 \mathrm{~L}$ & 3 & 0.06 & $2 \mathrm{R}$ \\
\hline Emericella & 190 & 1.80 & $15 \mathrm{H}$ & 153 & 1.64 & $13 \mathrm{H}$ & 53 & 0.56 & $7 \mathrm{M}$ & 47 & 1.01 & $7 \mathrm{M}$ \\
\hline E. dentata & 15 & 0.14 & $3 \mathrm{~L}$ & 9 & 0.10 & $3 \mathrm{~L}$ & & & & & & \\
\hline E. nidulans & & & & 3 & 0.03 & $1 \mathrm{R}$ & 2 & 0.02 & $2 \mathrm{R}$ & 1 & 0.02 & $1 \mathrm{R}$ \\
\hline E. quadrilineata & 1 & 0.01 & $1 \mathrm{R}$ & 2 & 0.02 & $2 \mathrm{R}$ & 3 & 0.03 & $3 \mathrm{~L}$ & 1 & 0.02 & $1 \mathrm{R}$ \\
\hline E. rugulosa & 1 & 0.01 & $1 \mathrm{R}$ & 2 & 0.02 & $1 \mathrm{R}$ & & & & & & \\
\hline E. stella-maris & 28 & 0.27 & $2 \mathrm{R}$ & 8 & 0.09 & $4 \mathrm{~L}$ & & & & & & \\
\hline
\end{tabular}




\begin{tabular}{|c|c|c|c|c|c|c|c|c|c|c|c|c|}
\hline & \multicolumn{5}{|c|}{ Citrus } & \multicolumn{7}{|c|}{ Grapevine } \\
\hline & \multicolumn{2}{|c|}{ DYM } & \multicolumn{4}{|c|}{ DRBC } & \multicolumn{2}{|c|}{ DYM } & \multicolumn{4}{|c|}{ DRBC } \\
\hline & $\mathrm{CFU}$ & $\% \mathrm{CFU}$ & $\mathrm{F} \& \mathrm{O}$ & $\mathrm{CFU}$ & $\% \mathrm{CFU}$ & $\mathrm{F} \& \mathrm{O}$ & $\mathrm{CFU}$ & $\% \mathrm{CFU}$ & $\mathrm{F} \& \mathrm{O}$ & $\mathrm{CFU}$ & $\% \mathrm{CFU}$ & $\mathrm{F} \& \mathrm{O}$ \\
\hline E. variecolor & 144 & 1.36 & $13 \mathrm{H}$ & 117 & 1.25 & $12 \mathrm{H}$ & 46 & 0.48 & $4 \mathrm{~L}$ & 40 & 0.86 & $5 \mathrm{M}$ \\
\hline Emericella sp. & & & & 11 & 0.12 & $2 \mathrm{R}$ & 2 & 0.02 & $1 \mathrm{R}$ & 3 & 0.06 & $1 \mathrm{R}$ \\
\hline Eurotium amstelodami & 2 & 0.02 & $2 \mathrm{R}$ & 5 & 0.05 & $1 \mathrm{R}$ & 1 & 0.01 & $1 \mathrm{R}$ & & & \\
\hline Fennellia flavipes & 6 & 0.06 & $2 \mathrm{R}$ & 4 & 0.04 & $2 \mathrm{R}$ & & & & 1 & 0.02 & $1 \mathrm{R}$ \\
\hline Fusarium & 308 & 2.92 & $18 \mathrm{H}$ & 114 & 1.22 & $13 \mathrm{H}$ & 159 & 1.67 & $15 \mathrm{H}$ & 39 & 0.84 & $8 \mathrm{M}$ \\
\hline F. babinda & 3 & 0.03 & $2 \mathrm{R}$ & & & & 2 & 0.02 & $1 \mathrm{R}$ & 3 & 0.06 & $1 \mathrm{R}$ \\
\hline F. camptoceras & 17 & 0.16 & $3 \mathrm{~L}$ & 14 & 0.15 & $3 \mathrm{~L}$ & & & & & & \\
\hline F. chlamydosporum & & & & & & & 2 & 0.02 & $2 \mathrm{R}$ & & & \\
\hline F. circinatum & & & & & & & 3 & 0.03 & $2 \mathrm{R}$ & & & \\
\hline$F$. concolor & 28 & 0.27 & $9 \mathrm{H}$ & 10 & 0.11 & $5 \mathrm{M}$ & 4 & 0.04 & $1 \mathrm{R}$ & & & \\
\hline F. equiseti & 34 & 0.32 & $9 \mathrm{H}$ & 18 & 0.19 & $8 \mathrm{M}$ & 22 & 0.23 & $6 \mathrm{M}$ & & & \\
\hline F. konzum & & & & & & & 1 & 0.01 & $1 \mathrm{R}$ & 1 & 0.02 & $1 \mathrm{R}$ \\
\hline F. oxysporum & & & & 3 & 0.03 & $2 \mathrm{R}$ & 1 & 0.01 & $1 \mathrm{R}$ & & & \\
\hline F. proliferatum & & & & & & & 2 & 0.02 & $2 \mathrm{R}$ & 20 & 0.43 & $1 \mathrm{R}$ \\
\hline F. pseudonygami & & & & & & & 1 & 0.01 & $1 \mathrm{R}$ & 2 & 0.04 & $1 \mathrm{R}$ \\
\hline$F$. semitectum & 201 & 1.90 & $18 \mathrm{H}$ & 52 & 0.56 & $11 \mathrm{H}$ & 116 & 1.22 & $12 \mathrm{H}$ & 8 & 0.17 & $5 \mathrm{M}$ \\
\hline F. solani & 15 & 0.14 & $3 \mathrm{~L}$ & 11 & 0.12 & $2 \mathrm{R}$ & & & & 4 & 0.09 & $2 \mathrm{R}$ \\
\hline F. subglutinans & & & & 2 & 0.02 & $1 \mathrm{R}$ & 3 & 0.03 & $3 R$ & 1 & 0.02 & $1 \mathrm{R}$ \\
\hline F. verticillioides & 9 & 0.09 & $4 \mathrm{~L}$ & 4 & 0.04 & $2 \mathrm{R}$ & 1 & 0.01 & $1 \mathrm{R}$ & & & \\
\hline Humicola & 24 & 0.23 & $8 \mathrm{M}$ & 30 & 0.32 & $11 \mathrm{H}$ & 8 & 0.08 & $4 \mathrm{~L}$ & 1 & 0.02 & $1 \mathrm{R}$ \\
\hline H. fuscoatra & 23 & 0.22 & $8 \mathrm{M}$ & 30 & 0.32 & $11 \mathrm{H}$ & 7 & 0.07 & $3 \mathrm{~L}$ & 1 & 0.02 & $1 \mathrm{R}$ \\
\hline H. grisea & 1 & 0.01 & $1 \mathrm{R}$ & & & & 1 & 0.01 & $1 \mathrm{R}$ & & & \\
\hline Memmnoniella echinata & 1 & 0.01 & $1 \mathrm{R}$ & 1 & 0.01 & $1 \mathrm{R}$ & & & & & & \\
\hline Microascus brevicaulis & 11 & 0.10 & $4 \mathrm{~L}$ & 5 & 0.05 & $1 \mathrm{R}$ & 1 & 0.01 & $1 \mathrm{R}$ & 3 & 0.06 & $1 \mathrm{R}$ \\
\hline Microdochium dimerum & & & & & & & 15 & 0.16 & $1 \mathrm{R}$ & 5 & 0.11 & $2 \mathrm{R}$ \\
\hline Mortierella alpina & 3 & 0.03 & $2 \mathrm{R}$ & 1 & 0.01 & $1 \mathrm{R}$ & & & & & & \\
\hline Mucor & 9 & 0.09 & $6 \mathrm{M}$ & 12 & 0.13 & $3 \mathrm{~L}$ & & & & 1 & 0.02 & $1 \mathrm{R}$ \\
\hline M. circinelloides & 4 & 0.04 & $3 \mathrm{~L}$ & 2 & 0.02 & $1 \mathrm{R}$ & & & & & & \\
\hline M. hiemalis & 4 & 0.04 & $3 \mathrm{~L}$ & 10 & 0.11 & $3 \mathrm{~L}$ & & & & 1 & 0.02 & $1 \mathrm{R}$ \\
\hline Myrothecium & 113 & 1.07 & $12 \mathrm{H}$ & 69 & 0.74 & $12 \mathrm{H}$ & 46 & 0.48 & $9 \mathrm{H}$ & 46 & 0.99 & $5 \mathrm{M}$ \\
\hline M. roridum & 4 & 0.04 & $2 \mathrm{R}$ & 12 & 0.13 & $3 \mathrm{~L}$ & 8 & 0.08 & $3 \mathrm{~L}$ & & & \\
\hline M. verrucaria & 109 & 1.03 & $11 \mathrm{H}$ & 57 & 0.61 & $11 \mathrm{H}$ & 38 & 0.40 & $7 \mathrm{M}$ & 46 & 0.99 & $5 \mathrm{M}$ \\
\hline Neosartorya fumigata & & & & & & & 4 & 0.04 & $3 \mathrm{~L}$ & & & \\
\hline Nigrospora oryzae & 8 & 0.08 & $4 \mathrm{~L}$ & 5 & 0.05 & $3 \mathrm{~L}$ & 8 & 0.08 & $4 \mathrm{~L}$ & 4 & 0.09 & $3 \mathrm{~L}$ \\
\hline Paecillium lilacinum & & & & & & & 44 & 0.46 & $1 \mathrm{R}$ & 53 & 1.14 & $1 \mathrm{R}$ \\
\hline Penicillium & 1307 & 12.38 & $18 \mathrm{H}$ & 1868 & 19.99 & $16 \mathrm{H}$ & 317 & 3.34 & $16 \mathrm{H}$ & 256 & 5.51 & $10 \mathrm{H}$ \\
\hline P. adametzioides & 95 & 0.90 & $1 \mathrm{R}$ & 35 & 0.37 & $1 \mathrm{R}$ & & & & 1 & 0.02 & $1 \mathrm{R}$ \\
\hline P. aurantiogriseum & 116 & 1.10 & $3 \mathrm{~L}$ & 245 & 2.62 & $3 \mathrm{~L}$ & & & & & & \\
\hline
\end{tabular}




\begin{tabular}{|c|c|c|c|c|c|c|c|c|c|c|c|c|}
\hline & \multicolumn{5}{|c|}{ Citrus } & \multicolumn{7}{|c|}{ Grapevine } \\
\hline & \multicolumn{2}{|c|}{ DYM } & \multicolumn{4}{|c|}{ DRBC } & \multicolumn{2}{|c|}{ DYM } & \multicolumn{4}{|c|}{ DRBC } \\
\hline & $\mathrm{CFU}$ & $\% \mathrm{CFU}$ & $\mathrm{F} \& \mathrm{O}$ & CFU & $\% \mathrm{CFU}$ & $\mathrm{F} \& \mathrm{O}$ & $\mathrm{CFU}$ & $\% \mathrm{CFU}$ & $\mathrm{F} \& \mathrm{O}$ & $\mathrm{CFU}$ & $\% \mathrm{CFU}$ & $\mathrm{F} \& \mathrm{O}$ \\
\hline P. bilaii & 33 & 0.31 & $2 \mathrm{R}$ & & & & & & & & & \\
\hline P. brevicompactum & 34 & 0.32 & $5 \mathrm{M}$ & 57 & 0.61 & $3 \mathrm{~L}$ & 6 & 0.06 & $1 \mathrm{R}$ & 2 & 0.04 & $1 \mathrm{R}$ \\
\hline P. citrinum & 114 & 1.08 & $9 \mathrm{H}$ & 153 & 1.64 & $6 \mathrm{M}$ & 1 & 0.01 & $1 \mathrm{R}$ & 1 & 0.02 & $1 \mathrm{R}$ \\
\hline P. corylophilum & & & & 2 & 0.02 & $1 \mathrm{R}$ & & & & 1 & 0.02 & $1 \mathrm{R}$ \\
\hline P. crustosum & 1 & 0.01 & $1 \mathrm{R}$ & 3 & 0.03 & $1 \mathrm{R}$ & & & & & & \\
\hline P. cyaneum & 4 & 0.04 & $2 \mathrm{R}$ & & & & & & & & & \\
\hline P. duclauxii & 15 & 0.14 & $4 \mathrm{~L}$ & 14 & 0.15 & $3 \mathrm{~L}$ & 4 & 0.04 & $3 \mathrm{~L}$ & & & \\
\hline P. expansum & 2 & 0.02 & $1 \mathrm{R}$ & & & & 1 & 0.01 & $1 \mathrm{R}$ & & & \\
\hline P. funiculosum & 3 & 0.03 & $2 \mathrm{R}$ & 4 & 0.04 & $2 \mathrm{R}$ & & & & & & \\
\hline P. glabrum & & & & 116 & 1.24 & $2 \mathrm{R}$ & 1 & 0.01 & $1 \mathrm{R}$ & & & \\
\hline$P$. granulatum & & & & 4 & 0.04 & $1 \mathrm{R}$ & & & & 1 & 0.02 & $1 \mathrm{R}$ \\
\hline P. griseofulvum & 38 & 0.36 & $5 \mathrm{M}$ & 47 & 0.50 & $4 \mathrm{~L}$ & 1 & 0.01 & $1 \mathrm{R}$ & & & \\
\hline P. implicatum & 2 & 0.02 & $2 \mathrm{R}$ & & & & & & & & & \\
\hline P. islandicum & & & & 1 & 0.01 & $1 \mathrm{R}$ & 2 & 0.02 & $1 \mathrm{R}$ & & & \\
\hline P. italicum & & & & 2 & 0.02 & $2 \mathrm{R}$ & & & & & & \\
\hline P. olsonii & 796 & 7.54 & $9 \mathrm{H}$ & 957 & 10.24 & $11 \mathrm{H}$ & & & & & & \\
\hline P. oxalicum & 12 & 0.11 & $3 \mathrm{~L}$ & 12 & 0.13 & $4 \mathrm{~L}$ & 287 & 3.02 & $13 \mathrm{H}$ & 245 & 5.28 & $9 \mathrm{H}$ \\
\hline P. pinophilum & 2 & 0.02 & $2 \mathrm{R}$ & 6 & 0.06 & $3 \mathrm{~L}$ & 1 & 0.01 & $1 \mathrm{R}$ & & & \\
\hline P. puberulum & 18 & 0.17 & $3 \mathrm{~L}$ & 5 & 0.05 & $2 \mathrm{R}$ & & & & & & \\
\hline P. purpurogenum & 17 & 0.16 & $7 \mathrm{M}$ & 20 & 0.21 & $6 \mathrm{M}$ & 12 & 0.13 & $5 \mathrm{M}$ & 4 & 0.09 & $2 \mathrm{R}$ \\
\hline P. verrucosum & & & & 7 & 0.07 & $2 \mathrm{R}$ & & & & & & \\
\hline P. vulpinum & 3 & 0.03 & $2 \mathrm{R}$ & 62 & 0.66 & $1 \mathrm{R}$ & & & & & & \\
\hline Petromyces flavus (ana: Aspergillus flavus) & 19 & 0.18 & $9 \mathrm{H}$ & 29 & 0.31 & $7 \mathrm{M}$ & 13 & 0.14 & $5 \mathrm{M}$ & 11 & 0.24 & $9 \mathrm{H}$ \\
\hline Phoma & 91 & 0.86 & $14 \mathrm{H}$ & 46 & 0.49 & $6 \mathrm{M}$ & 171 & 1.80 & $13 \mathrm{H}$ & 63 & 1.36 & $8 \mathrm{M}$ \\
\hline P. epicoccina & 88 & 0.83 & $14 \mathrm{H}$ & 46 & 0.49 & $6 \mathrm{M}$ & 138 & 1.45 & $12 \mathrm{H}$ & 57 & 1.23 & $8 \mathrm{M}$ \\
\hline P. eupyrena & & & & & & & 32 & 0.34 & $2 \mathrm{R}$ & & & \\
\hline Phoma sp. & 3 & 0.03 & $2 \mathrm{R}$ & & & & 1 & 0.01 & $1 \mathrm{R}$ & & & \\
\hline Pleospora & 45 & 0.43 & $10 \mathrm{H}$ & 46 & 0.49 & $11 \mathrm{H}$ & 35 & 0.37 & $7 \mathrm{M}$ & 36 & 0.78 & $9 \mathrm{H}$ \\
\hline P. tarda & 43 & 0.41 & $10 \mathrm{H}$ & 45 & 0.48 & $11 \mathrm{H}$ & 35 & 0.37 & $7 \mathrm{M}$ & 36 & 0.78 & $9 \mathrm{H}$ \\
\hline Pochonia sp. & 2 & 0.02 & $2 \mathrm{R}$ & & & & & & & 1 & 0.02 & $1 \mathrm{R}$ \\
\hline Pseudonectria pachysandricola & 15 & 0.14 & $5 \mathrm{M}$ & 3 & 0,03 & $1 \mathrm{R}$ & & & & & & \\
\hline Quambalaria cyanescens & 8 & 0.08 & $1 \mathrm{R}$ & 3 & 0.03 & $1 \mathrm{R}$ & 10 & 0.11 & $2 \mathrm{R}$ & 2 & 0.04 & $2 \mathrm{R}$ \\
\hline Rhizopus oryzae & 7 & 0.07 & $7 \mathrm{M}$ & 1 & 0.01 & $1 \mathrm{R}$ & 25 & 0.26 & $10 \mathrm{H}$ & 23 & 0.49 & $3 \mathrm{~L}$ \\
\hline Sarcopodium araliae & & & & 2 & 0.02 & $2 \mathrm{R}$ & & & & & & \\
\hline Scytalidium sp. & & & & 2 & 0.02 & $1 \mathrm{R}$ & 1 & 0.01 & $1 \mathrm{R}$ & & & \\
\hline Setosphearia & 48 & 0.46 & $8 \mathrm{M}$ & 40 & 0.43 & $5 \mathrm{M}$ & 763 & 8.03 & $17 \mathrm{H}$ & 605 & 13.03 & $15 \mathrm{H}$ \\
\hline S. rostrata & 47 & 0.45 & $8 \mathrm{M}$ & 40 & 0.43 & $5 \mathrm{M}$ & 763 & 8.03 & $17 \mathrm{H}$ & 603 & 12.98 & $15 \mathrm{H}$ \\
\hline Stachybotrys chartarum & 14 & 0.13 & $3 \mathrm{~L}$ & 6 & 0.06 & $2 \mathrm{R}$ & 3 & 0.03 & $1 \mathrm{R}$ & & & \\
\hline
\end{tabular}




\begin{tabular}{|c|c|c|c|c|c|c|c|c|c|c|c|c|}
\hline & \multicolumn{5}{|c|}{ Citrus } & \multicolumn{7}{|c|}{ Grapevine } \\
\hline & \multicolumn{2}{|c|}{ DYM } & \multicolumn{4}{|c|}{ DRBC } & \multicolumn{2}{|c|}{ DYM } & \multicolumn{4}{|c|}{ DRBC } \\
\hline & $\mathrm{CFU}$ & $\% \mathrm{CFU}$ & $\mathrm{F} \& \mathrm{O}$ & $\mathrm{CFU}$ & $\% \mathrm{CFU}$ & $\mathrm{F} \& \mathrm{O}$ & $\mathrm{CFU}$ & $\% \mathrm{CFU}$ & $\mathrm{F} \& \mathrm{O}$ & $\mathrm{CFU}$ & $\% \mathrm{CFU}$ & $\mathrm{F} \& \mathrm{O}$ \\
\hline Stemphylium & 4 & 0.04 & $4 \mathrm{~L}$ & 23 & 0.25 & $5 \mathrm{M}$ & 11 & 0.12 & $2 \mathrm{R}$ & 71 & 1.53 & $6 \mathrm{M}$ \\
\hline S. sarciniforme & 1 & 0.01 & $1 \mathrm{R}$ & & & & 8 & 0.08 & $2 \mathrm{R}$ & 6 & 0.13 & $2 \mathrm{R}$ \\
\hline Stemphylium spp. & 3 & 0.03 & $3 \mathrm{~L}$ & 23 & 0.25 & $5 \mathrm{M}$ & 3 & 0.03 & $1 \mathrm{R}$ & 65 & 1.39 & $6 \mathrm{M}$ \\
\hline Talaromyces stipitatus & 1 & 0.01 & $1 \mathrm{R}$ & 1 & 0.01 & $1 \mathrm{R}$ & & & & 6 & 0.13 & $1 \mathrm{R}$ \\
\hline Toxicocladosporium irritans & 1 & 0.01 & $1 \mathrm{R}$ & 2 & 0.02 & $1 \mathrm{R}$ & & & & & & \\
\hline Trichoderma & 6 & 0.06 & $6 \mathrm{M}$ & 6 & 0.06 & $5 \mathrm{M}$ & & & & 4 & 0.09 & $3 \mathrm{~L}$ \\
\hline T. harzianum & 1 & 0.01 & $1 \mathrm{R}$ & 3 & 0.03 & $2 \mathrm{R}$ & & & & 1 & 0.02 & $1 \mathrm{R}$ \\
\hline T. paraceramosum & 3 & 0.03 & $3 \mathrm{~L}$ & 1 & 0.01 & $1 \mathrm{R}$ & & & & 3 & 0.06 & $2 \mathrm{R}$ \\
\hline T. reesei & 1 & 0.01 & $1 \mathrm{R}$ & 2 & 0.02 & $2 \mathrm{R}$ & & & & & & \\
\hline Ulocladium & & & & 2 & 0.02 & $2 \mathrm{R}$ & 19 & 0.20 & $2 \mathrm{R}$ & 8 & 0.17 & $2 \mathrm{R}$ \\
\hline U. atrum & & & & 1 & 0.01 & $1 \mathrm{R}$ & 19 & 0.20 & $2 \mathrm{R}$ & 8 & 0.17 & $2 \mathrm{R}$ \\
\hline Volutella sp. & 6 & 0.06 & $2 \mathrm{R}$ & 1 & 0.01 & $1 \mathrm{R}$ & & & & & & \\
\hline Yeasts & 414 & 3.92 & $16 \mathrm{H}$ & 125 & 1.34 & $14 \mathrm{H}$ & 557 & 5.86 & $15 \mathrm{H}$ & 70 & 1.51 & $7 \mathrm{M}$ \\
\hline Candida & 2 & 0.02 & $2 \mathrm{R}$ & 5 & 0.05 & $3 \mathrm{~L}$ & & & & & & \\
\hline C. catenulate & 2 & 0.02 & $2 \mathrm{R}$ & & & & & & & & & \\
\hline C. parapsilosis & & & & 5 & 0.05 & $3 \mathrm{~L}$ & & & & & & \\
\hline Cryptococcus & 66 & 0.63 & $9 \mathrm{H}$ & 47 & 0.50 & $7 \mathrm{M}$ & 58 & 0.62 & $10 \mathrm{H}$ & 37 & 0.79 & $6 \mathrm{M}$ \\
\hline C. albidus & 66 & 0.63 & $9 \mathrm{H}$ & 47 & 0.50 & $7 \mathrm{M}$ & 33 & 0.35 & $6 \mathrm{M}$ & 20 & 0.43 & $4 \mathrm{~L}$ \\
\hline C. laurentii & & & & & & & 17 & 0.18 & $4 \mathrm{~L}$ & 17 & 0.37 & $6 \mathrm{M}$ \\
\hline Debaryomyces & 20 & 0.19 & $4 \mathrm{~L}$ & 10 & 0.11 & $5 \mathrm{M}$ & 7 & 0.07 & $4 \mathrm{~L}$ & 2 & 0.04 & $1 \mathrm{R}$ \\
\hline D. hansenii & 15 & 0.14 & $3 \mathrm{~L}$ & 8 & 0.09 & $4 \mathrm{~L}$ & 3 & 0.03 & $2 \mathrm{R}$ & 2 & 0.04 & $1 \mathrm{R}$ \\
\hline D. pseudopolymorphus & 5 & 0.05 & $2 \mathrm{R}$ & 2 & 0.02 & $1 \mathrm{R}$ & 4 & 0.04 & $2 \mathrm{R}$ & & & \\
\hline Geotrichum & 2 & 0.02 & $1 \mathrm{R}$ & 2 & 0.02 & $2 \mathrm{R}$ & & & & & & \\
\hline G. citri-aurantii & 2 & 0.02 & $1 \mathrm{R}$ & 1 & 0.02 & $1 \mathrm{R}$ & & & & & & \\
\hline Hanseniaspora occidentalis & 24 & 0.23 & $2 \mathrm{R}$ & 2 & 0.02 & $1 \mathrm{R}$ & & & & & & \\
\hline Issatchenkia orientalis & 4 & 0.04 & $1 \mathrm{R}$ & 3 & 0.03 & $2 \mathrm{R}$ & 1 & 0.01 & $1 \mathrm{R}$ & & & \\
\hline Melanopsichium pennsylvanicum & 2 & 0.02 & $1 \mathrm{R}$ & 1 & 0.01 & $1 \mathrm{R}$ & & & & & & \\
\hline Pichia & 18 & 0.17 & $2 \mathrm{R}$ & 7 & 0.07 & $1 \mathrm{R}$ & 53 & 0.57 & $3 \mathrm{~L}$ & 8 & 0.17 & $3 \mathrm{~L}$ \\
\hline P. guilliermondii & 17 & 0.16 & $1 \mathrm{R}$ & 7 & 0.07 & $1 \mathrm{R}$ & 53 & 0.57 & $3 \mathrm{~L}$ & 8 & 0.17 & $3 \mathrm{~L}$ \\
\hline Pseudozyma & 2 & 0.02 & $1 \mathrm{R}$ & 9 & 0.09 & $1 \mathrm{R}$ & 5 & 0.05 & $1 \mathrm{R}$ & & & \\
\hline P. hubeinsis & 2 & 0.02 & $1 \mathrm{R}$ & 9 & 0.09 & $1 \mathrm{R}$ & & & & & & \\
\hline Rhodosporidium paludigenum & 93 & 0.88 & $6 \mathrm{M}$ & 12 & 0.13 & $4 \mathrm{~L}$ & & & & & & \\
\hline Rhodotorula & 63 & 0.59 & $10 \mathrm{H}$ & 18 & 0.19 & $5 \mathrm{M}$ & 428 & 4.58 & $9 \mathrm{H}$ & 18 & 0.39 & $3 \mathrm{~L}$ \\
\hline R. aurantiaca & 2 & 0.02 & $2 \mathrm{R}$ & & & & & & & & & \\
\hline R. glutinis & 12 & 0.11 & $4 \mathrm{~L}$ & 2 & 0.02 & $1 \mathrm{R}$ & 378 & 4.05 & $6 \mathrm{M}$ & 16 & 0.34 & $3 \mathrm{~L}$ \\
\hline R. mucilaginosa & 49 & 0.46 & $5 \mathrm{M}$ & 16 & 0.17 & $4 \mathrm{~L}$ & 50 & 0.54 & $4 \mathrm{~L}$ & 2 & 0.04 & $1 \mathrm{R}$ \\
\hline Sporidiobolus & 119 & 1.14 & $5 \mathrm{M}$ & 5 & 0.05 & $3 \mathrm{~L}$ & 5 & 0.05 & $3 \mathrm{~L}$ & 2 & 0.04 & $1 \mathrm{R}$ \\
\hline S. ruineniae & 119 & 1.14 & $5 \mathrm{M}$ & 5 & 0.05 & $3 \mathrm{~L}$ & 4 & 0.04 & $2 \mathrm{R}$ & 2 & 0.04 & $1 \mathrm{R}$ \\
\hline
\end{tabular}




\begin{tabular}{|c|c|c|c|c|c|c|c|c|c|c|c|c|}
\hline & \multicolumn{5}{|c|}{ Citrus } & \multicolumn{7}{|c|}{ Grapevine } \\
\hline & \multicolumn{2}{|c|}{ DYM } & \multicolumn{4}{|c|}{ DRBC } & \multicolumn{2}{|c|}{ DYM } & \multicolumn{4}{|c|}{ DRBC } \\
\hline & $\mathrm{CFU}$ & $\% \mathrm{CFU}$ & $\mathrm{F} \& \mathrm{O}$ & CFU & $\% \mathrm{CFU}$ & $\mathrm{F} \& \mathrm{O}$ & $\mathrm{CFU}$ & $\% \mathrm{CFU}$ & $\mathrm{F} \& \mathrm{O}$ & $\mathrm{CFU}$ & $\% \mathrm{CFU}$ & $\mathrm{F} \& \mathrm{O}$ \\
\hline Total CFUs & 10555 & 100 & $18 \mathrm{H}$ & 9343 & 100 & $18 \mathrm{H}$ & 9505 & 100 & $18 \mathrm{H}$ & 4644 & 100 & $18 \mathrm{H}$ \\
\hline No. of genera (83) & \multicolumn{3}{|c|}{59} & \multicolumn{3}{|l|}{58} & \multicolumn{3}{|l|}{46} & \multicolumn{3}{|l|}{44} \\
\hline No. of species + varieties $(218+3)$ & \multicolumn{2}{|c|}{$139+2$ var. } & & \multicolumn{3}{|c|}{$135+2$ var. } & \multicolumn{2}{|c|}{$108+2$ var. } & & \multicolumn{3}{|c|}{$89+1$ variety } \\
\hline
\end{tabular}

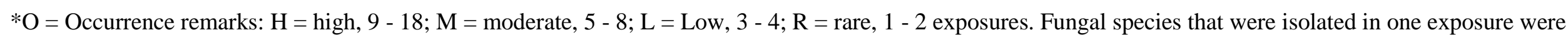
omitted from the table (but their frequency of occurrence and counts were included in statistical analysis).

Table 4a NMDS statistical analysis of data obtained from the frequency of occurrence of genera and species recovered from the two habitats on the 2 isolation media.

\begin{tabular}{|c|c|c|c|c|c|}
\hline \multirow[t]{2}{*}{ Parameter } & & \multicolumn{2}{|l|}{ Citrus air } & \multicolumn{2}{|c|}{ Grapevine air } \\
\hline & & DYM & DRBC & DYM & DRBC \\
\hline \multirow[t]{7}{*}{ Genera vs Habitats } & $\mathrm{S}$ & 59 & 58 & 46 & 44 \\
\hline & $\mathrm{E}$ & 0.900 & 0.892 & 0.889 & 0.890 \\
\hline & $\mathrm{H}$ & 3.669 & 3.623 & 3.404 & 3.369 \\
\hline & $\mathrm{D}$ & 0.9675 & 0.9644 & 0.9577 & 0.9551 \\
\hline & $\mathrm{n}$ & 73 & 67 & & \\
\hline & No of genera in one plant & 27 & 23 & 14 & 9 \\
\hline & No of genera in 2 plants & 32 & 35 & 32 & 35 \\
\hline \multirow[t]{7}{*}{ Species vs Habitats } & $\mathrm{S}$ & 141 & 137 & 110 & 90 \\
\hline & $\mathrm{E}$ & 0.917 & 0.922 & 0.909 & 0.904 \\
\hline & $\mathrm{H}$ & 4.540 & 4.537 & 4.274 & 4.066 \\
\hline & $\mathrm{D}$ & 0.9854 & 0.9854 & 0.9807 & 0.9764 \\
\hline & $\mathrm{n}$ & 185 & 164 & & \\
\hline & No of species in one plant & 75 & 74 & 44 & 27 \\
\hline & No of species in 2 plants & 66 & 63 & 66 & 63 \\
\hline
\end{tabular}

$\mathrm{S}=$ Genera and species richness, $\mathrm{E}=$ Evenness of frequency of occurrence of genera or species in the samples, $\mathrm{H}=\mathrm{Shannon}$ 's index of genera or species diversity, $\mathrm{H}=-\sum_{i}^{s} \mathrm{Pi} \ln \mathrm{Pi}, \mathrm{D}=$ Simpson's index of genera or species diversity, $\mathrm{D}=-\sum_{i}^{s} \mathrm{Pi}^{2}$. 
Table 4b NMDS statistical analysis of data obtained from the total counts of all species (CFUs) recovered from the two habitats on the 2 isolation media.

\begin{tabular}{llllll}
\hline Parameter & & Citrus air & \multicolumn{2}{c}{$\begin{array}{l}\text { Grapevine air } \\
\text { DYM }\end{array}$} & DRBC \\
\hline Genera vs Habitats & E & DYM & DRBC & DYM \\
& H & 0.415 & 0.384 & 0.528 & 0.543 \\
Species vs Habitats & D & 1.692 & 1.561 & 2.023 & 2.054 \\
& E & 0.6374 & 0.6536 & 0.7866 & 0.8155 \\
& H & 0.467 & 0.501 & 0.559 & 0.618 \\
& D & 2.314 & 2.463 & 2.618 & 2.780 \\
& Total counts of all species & 0.6969 & 0.7596 & 0.8406 & 0.8892 \\
& D & 10555 & 9343 & 9505 & 4644 \\
\hline
\end{tabular}

Emericella (10 species and 3 unidentified) was isolated in high frequency from citrus air and in moderate frequency from grapevine air. Emericella variecolor was the most common species in citrus and grapevine plantations. It is worthy to mention that the isolation of E. stellamaris in this study from citrus air is a second world record after its first description in 2008.

Myrothecium (represented by $M$. verrucaria, $M$. roridum and Myrothecium sp.) was recovered in high frequency from the air of both plantations. $M$. verrucaria was the most common species followed by $M$. roridum in both plantations.

Botryodiplodia (B. theobromae) was recorded in high or moderate frequency from citrus air and in rare frequency from grapevine air. On contrary, Setosphaeria (S. monoceras, S. rostrata and S. pedicillata) was isolated in high frequency from grapevine air and in moderate frequency from citrus air, with $S$. rostrata being the main species, whereas $S$. monoceras was isolated from citrus air and S. pedicillata from grapevine air only.

Phoma (5 species and 1 unidentified) and Pleospora (P. allii, P. herbarum and P. tarda, teleomorphs of Stemphylium vesicarium, $S$. herbarum and $S$. botryosum respectively) were recorded in high frequency in the air of both plantations. The most common Phoma species was $P$. epicoccina (= Epicoccum nigrum) in both plantations, followed by $P$. eupyrena in grapevine plantations, while Pleospora tarda was the most frequent species isolated from both plantations and $P$. herbarum was more common in citrus plantations than grapevine.

Trichoderma (9 species and 1 unidentified) was encountered in moderate frequency from citrus air and in low frequency from grapevine air. The most common species were T. harzianum and T.reesei in citrus plantations and T. paracemosum in both plantations.

Rhizopus ( $R$. oryzae) was recorded in high frequency from grapevine air and in moderate frequency from citrus air while Mucor (4 species with $M$. circinelloides being the most common in both air followed by $M$. hiemalis) was recovered in moderate frequency from citrus air and rare from grapevine plantations. Humicola (3 species) was isolated in high frequency from citrus air and in low frequency from grapevine air. H. fuscoatra was more common than H. grisea in both plantations. Stachybotrys (S. chartarum and synnematous species of Stachybotrys) was recorded in rare frequency from the air of grapevine while in low frequency from citrus air.

Nigrospora ( $N$. oryzae and $N$. sphaerica) was identified in low frequency from the air of both plantations. $N$. oryzae was more frequent than $N$. sphaerica.

Acremonium ( 9 species and 1 unidentified) was isolated in low frequency from grapevine air and in rare frequency from citrus air. A. strictum was the most common species followed by $A$. fusidioides and A. hyalinulum. On contrary, Microascus (M. brevicaulis and M. manginii, teleomorphs of Scopulariopsis. brevicaulis and S. candida respectively) was isolated in low frequency from citrus air and in rare frequency from grapevine air.

Yeast fungi represented by 15 genera and 26 species were isolated from the air of both plantations. They showed their peak of total propagules caught from the air of citrus plantations in December on both media and from grapevine plantations in October and April on DYM and DRBC respectively, while their trough occurred regularly in April on both media in the air of citrus and in June and December on DYM and DRBC respectively in grapevine air. 
Two genera of yeast fungi were encountered in high frequency on one medium and moderate or low on the other medium in the air of both plantations and these were Cryptoccocus ( 4 species) and Rhodotorula (3 species). On the other hand, two genera were recovered in moderate or low frequency from the air of citrus and low or rare frequency from grapevine air and these were Debaryomyces (2 species) and Sporidiobolus (S. ruineniae). Some yeast genera were recovered only from the air of citrus (Ambrosiozyma, Candida, Geotrichum, Hanseniaspora, Rhodosporidium and Melanopsichium) while Sporobolomyces and Trichosporon from grapevine plantations only (Table 2).

Cryptococcus was recovered in high or moderate frequency in both plantations. It contributed $0.50 \%-0.63 \%$ of total fungi in citrus air and $0.62 \%-0.79 \%$ in grapevine air. $C$. albidus was recovered in high or moderate frequency while it was recorded in moderate or low frequency in grapevine air. C. carnescence, C. flavescens and C. laurentii were isolated from grapevine air only.

Rhodotorula was recorded in high or moderate frequency in citrus air while in high or low frequency in grapevine air, constituting $0.59 \%-0.19 \%$ and $4.58 \%-0.39 \%$ of total fungi respectively. $R$. mucilaginosa was more common in citrus air than $R$. glutinis while the reverse occurred in grapevine air. $R$. aurantiaca was recorded in rare frequency from citrus air only.

Candida was isolated in citrus air in low or rare frequency, but was missed in grapevine air. Candida catenulata was recovered on DYM while C. parapsilosis was isolated on DRBC.

Debaryomyces was recorded in citrus air in moderate or low frequency while in low or rare frequency in grapevine air, contributing minute percentage counts $(0.63 \%-0.50 \%$ of total fungi) in citrus air and (0.35\%-0.43\%) in grapevine air. D. hansenii was recovered in low frequency on both media in citrus air and in rare frequency in grapevine air. D. pseudopolymorphus was recorded in rare frequency in the air of both plantations.

\section{Statistical analysis using NMDS method}

Data presented in table (4a) reveal that the evenness of actual presence (frequency of occurrence) of genera and species was slightly higher in citrus air than in grapevine air on both media. Shannon's index $(\mathrm{H})$ indicates that the diversity of genera and species was relatively higher in citrus than those in grapevine air. Simpson's index (D) indicates that the genera and species diversity was more proportionally important in citrus than in grapevine air. When the counts of all species were considered instead of frequency for calculations (Table $4 \mathrm{~b})$, Shannon's index $(\mathrm{H})$ indicates that the diversity of genera and species was relatively higher in grapevine than those in citrus air and Simpson's index (D) indicates that the genera and species diversity was more proportionally important in grapevine than in citrus air.

\section{Discussion}

A total of 176 and 138 species and 3 varieties belonging to 66 and 58 genera were recovered respectively, from the air of citrus and grapevine plantations ( 3 farms in the six trips) bimonthly during the period from April 2008 to February 2009. Citrus plant has an advantage of being taller tree with dense evergreen foliage that may affect most environments around it more positively than grapevine. The filamentous fungi constituted the greatest part of propagules (96.08\% and $94.03 \%$ on DYM and $98.67 \%$ and $98.49 \%$ on DRBC) while yeasts contributed 3.92\% and $5.97 \%$ and $1.33 \%$ and $1.51 \%$ of total fungi in the two plantations respectively.

DYM regularly sustained a broader spectrum of genera and species in the air of the two plantations then DRBC (139 species + 2 varieties and 59 genera in citrus versus 108 species +2 varieties and 46 genera in grapevine on DYM) and denser number of CFUs (20089 versus 13987).

The most common genera of filamentous fungi were Cladosporium, Penicillium, Aspergillus, Fusarium, Alternaria, Emericella, Myrothecium, Cochliobolus, Phoma, Pleospora, Setosphaeria, Botryodiplodia, Humicola, Petromyces and Rhizopus.

Cladosporium was the most dominant fungus in the air of both plants, yielding considerably higher numbers of propagules in citrus plantations than in grapevine. Its count peak was regularly 
recorded in winter/spring months, while its trough was registered in summer/autumn months in the air of both plantations. $C$. cladosporioides was the most common species, contributing about half of total fungi in the air of citrus and about one-fourth to one-third in the air of grapevine. $C$. sphaerospermum came second in the air of both plantations, while $C$. herbarum was isolated only from the air of grapevine. In several aeromycological studies and in agreement with the current results, Cladosporium was considered as one of most abundant genera, if not, the most, abundant one reported all over the world such as Egypt (Moubasher \& Moustafa 1974, El-Sherbeny 1987, Moubasher 1993, Ismail et al. 2002), Hellwan area, Egypt (Abdel Hameed et al. 2009), banana field in Qena (El-Said \& Abdel-Hafez 1995), Uganda (Ismail et al. 1999), USA (Lacey 1981), Porto (Oliveira et al. 2005), Yemen (El-Essawy et al. 1992), Doha, Qatar (Al-Subai 2002), Eskisehir and Afyonkarahisar, Turkey (Asan et al. 2004, O"zkara et al. 2007), Sari and Zanjan, Iran (Hedayati et al. 2005, Nourian et al. 2007), and Amman and Zarqa area, Jordan (Shaheen 1992, Abu-Dieyeh et al. 2010). Cladosporium spores are the dominant aerospora in hot climates (Takahashi 1997, Sen \& Asan 2001, Al-Subai 2002, El-Morsy 2006). Cladosporium spores contributed the highest number $(26.87 \%$ to the total sugarcane fields aerspora) in India (Ahire et al. 2010), of aerospora in groundnut fields (47.32\%) in Visakhapatnam, India (Mallaiah \& Rao 1990), as well as of the aerospora $(65.7 \%)$ in Hong Kong (Turner 1966). C. cladosporioides was the dominant species recorded of fungal aerospora in a coastal sandy belt of Orissa, India (Panda et al. 2009). According to Moubasher (1995) and Soliman (2012) comparison between the composition of air-borne fungal spores and soil mycobiota, and between air-borne spores and phyllosphere fungi reveal basic similarity between air- and phyllosphere borne fungi, which suggest that air-borne spores are mainly contributed by fungi developing on plant surface. This lends further support to Gregory's (1973) hypothesis that air-borne spores are basically a contribution from the vegetation rather than from the soil itself. This observation may show that some fungi such as Cladosporium spp. are strong competitive colonizers of the aerial plant materials unlike the case in soil whereas they are weak competitive colonizers of plant materials in soil, and hence they are more prevalent in air than in soil.

Soliman (2012) could deduce four patterns of correlation between the dominance (counts) of certain groups of fungi and the different studied sources in citrus and grapevine plantations, namely, soil air, phyllosphere, phylloplane, carposphere, carpoplane, and fruit juice. In soil pattern, the hyaline fungi e.g. Penicillium, Aspergillus, and Fusarium predominated over dark-coloured one e.g. Cladosporium, Alternaria, Bipolaris, and Exerohilum. In the air, phyllosphere and phylloplane pattern, the reverse occurs and the the dematiacous fungi outnumbered the hyaline ones. Soil is heavily populated by microorganisms and competition for existence is very severe. But the fungi are relatively protected from the injurious effects of atmospheric conditions: high light intensity, and deep diurnal fluctuations of temperature and humidity. These conditions may selectively favour the hyaline fungi over the dark-coloured (melanin-containing) fungi. In the air, phyllosphere and phylloplane pattern the environmental factors are markedly different which may induce selective effects for the advantage of the melanin-containing fungi over the hyaline ones. Melanin may protect fungi against stresses of solar UV and IR radiation.

Aspergillus contributed relatively higher numbers of propagules in the air of grapevine plantations (one-fourth of total fungi) than those from citrus plantations $(11.47 \%-13.98 \%)$. Its peak was regularly recorded in August in both plants. Species of Aspergillus belonging to section Nigri were the most common in the air of grapevine contributing relatively large proportions of Aspergillus count while, A. ochraceus was the most common species in the air of citrus, contributing large numbers of propagules. The dominance of these aspergilli agree with those obtained from the air of citrus plantations (Naim 1967, Moubasher et al. 1971), vineyard (Díaz et al. 2009), tomato plantations (Lohare et al. 2009), air at Assuit (Moubasher \& Moustafa 1974, AbuEl-Souod 1974), air at Qena (Moubasher et al. 1981, 1982) and air at Zagazig (El-Sherbeny 1987).

Alternaria was the second most frequent (behind Aspergillus) in grapevine air and the third behind Aspergillus and Cladosporium in citrus air, yielding considerably higher numbers of propagules in the air of grapevine plantations than those from citrus plantations. Its peak was 
recorded in winter months in the air of both plantations. Alternaria was isolated from the air of tomato plantations at Udgir, Latur District, India (Lohare et al. 2009), of the tea plantation area of Cachar District, Assam (Dutta et al. 2010), Brisbane, Queensland (Rees 1964), Hong Kong (Turner 1966) and Tulsa campus, USA (Levetin \& Dorsey 2006), at Modinagar, India (Bhati \& Guar 1979), at Zarqa area, Jordan (Abu-Dieyeh et al. 2010). A. alternata which was the most common species in the air of both plantations was also the dominant species in the air of citrus plantations (Moubasher et al. 1971) and the air of Assuit (Moubasher \& Moustafa 1974) in Egypt, and Coastal Belt of Orissa in India (Panda et al. 2009).

Fusarium was of high frequency in citrus and in high or moderate frequency in grapevine air, yielding small numbers of propagules $(0.84 \%-2.92 \%$ of total fungi in both plantations. Its peak was regularly recorded in June in the air of citrus plantations on both media but irregularly in the air of grapevine plantations, in October and August on DYM and DRBC respectively. Nine species were identified in the air of citrus plantations and 11 species in grapevine air of which $F$. semitectum was the most common species. $F$. equiseti and $F$. concolor were recorded in high or moderate frequency in citrus air, while in grapevine air $F$. equiseti was isolated in moderate frequency and $F$. concolor in rare frequency. F. camptoceras and $F$. torulosum were isolated from citrus air only while $F$. chlamydosporum, $F$. circinatum, $F$. konzum, $F$. proliferatum, $F$. pseudonygami and F. scripi were isolated from grapevine air only. Fusarium dominated in the air of citrus plantations in Upper Egypt (Moubasher et al. 1971), tea plantation area of Cachar District, Assam, India (Dutta et al. 2010), air of Coastal Belt of Orissa, India (Panda et al. 2009), and atmosphere at Modinagar, India (Bhati \& Guar 1979). Fusarium was also identified from the aerospora of Tulsa campus, USA (Levetin \& Dorsey 2006) and from the air of Hong Kong (Turner 1966). It is worthmentioning that Fusarium was the most common genus in the soil of both plants. $F$. solani was the most common in the soil of both plants followed by $F$. semitectum in citrus plants, and by $F$. oxysporum and $F$. babinda in grapevine plants (Soliman 2012).

Penicillium was one of the most common genera contributing noticeably higher numbers of propagules in the air of citrus than those from the air of grapevine. Its peak was recorded in February and April on DYM and DRBC respectively in citrus air and in August on both media in grapevine air. In citrus air, Penicillium olsonii was the most common species while it was missed in grapevine air and $P$. citrinum was also isolated in high or moderate frequency from citrus air but was recorded in rare frequency from grapevine air. On the other hand, P. oxalicum was the most common species in the air of grapevine, contributing relatively the largest proportions of Penicillium counts while it was recorded in low frequency in citrus air. Fifteen species of Penicillium were recorded in citrus air while two species in grapevine only. Penicillium dominated in the air of Assuit (Moubasher and Moustafa 1974), the eastern desert of Egypt (Ismail et al. 2002), Hellwan area, Egypt (Abdel Hameed et al. 2009), tea plantation in Barak Valley, Assam, India (Dutta et al. 2010), Hong Kong (Turner 1966), Mondiagar, Uttar Pradesh, India (Bhati \& Gaur 1979), Amman and Zarqa area, Jordan (Shaheen 1992, Abu-Dieyeh et al. 2010), Kuwait (Moustafa \& Kamel 1979), Saudi Arabia (Abdel-Hafez 1984), and Qatar (Al-Subai 2002). Penicillium was recorded in the air of Tulsa campus, USA (Levetin \& Dorsey 2006). Penicillium italicum and $P$. digitatum which are the main incitants of citrus rot were isolated from the air of orange groves and packing sheds (Ben-Meir-Glueck 1952) and air of citrus fruit packing houses, Israel (Barkai-Gollan 1961). Penicillium expansum and $P$. solitum were the most prevalent species while Penicillium digitatum was not common in the atmosphere of orchards and storage rooms of apples in Picardy and Pays de la Loire, France (Amiri \& Bompeix 2005).

Cochliobolus (2 species) and Myrothecium (represented by M. verrucaria, M. roridum and Myrothecium sp.) were recorded in high frequency in the air of both plantations. C. lunatus and $M$. verrucaria followed by $C$. australiensis and $M$. roridum were the most prevalent species. Curvularia (4 species) was isolated in low frequency from the air of both plantations. Cochliobolus lunatus (=Curvularia lunata) was one of the most dominant species of the aerospora in a coastal sandy belt of Orissa, India (Panda et al. 2009). Curvularia, Pithomyces, and Memnoniella were 
isolated from the aerospora in Jamaican banana plantations (Meredith 1961). Curvularia, Epicoccum, Pithomyces, Drechslera and Nigrospora were identified from Ulmus americana and Quercus palustris leaves, USA (Levetin \& Dorsey 2006). Drechslera (with D. spicifera, D. halodes and $D$. hawaiiensis being the most prevalent), Curvularia (C. lunata), Phoma (P. humicola, P. herbarum) and Chaetomium (C. globosum) were the most frequent genera in the Qat (Catha edulis) phyllosphere, Yemen (Alhubaishi \& Abdel-Kader 1991). M. verrucaria was isolated from the air at Assuit (Moubasher \& Moustafa 1974).

Emericella (10 species and 3 unidentified) and Botryodiplodia (B. theobromae) were recorded in high frequency from citrus air and in moderate frequency from grapevine air. Emericella variecolor was the most common species in citrus and grapevine plantations. It is worthy to mention that the isolation of E. stella-maris in this study from citrus plantations is the second world record (Moubasher et al. 2013) after its first description in 2008 in the Mediterranean region from Eucalyptus leaf litter in Tunisia and hypersaline saltern water in Slovenia (Zalar et al. 2008). Emericella species were isolated from cocoa beans (Sanchez-Harvas et al. 2008). E. variecolor was isolated from the air of Assiut (Moubasher \& Moustafa 1974). B. theobromae was isolated from decayed papaya fruits (Bagwan 2011) and from soil, Egypt (Mazen 1973). It was recorded as causal agent of mango and banana fruit-rot (El-Helaly et al. 1966).

Phoma (6 species with P. epicoccina being the most common) and Pleospora (3 species with $P$. tarda the most frequent) were recorded in high frequency in the air of both plantations. Phoma sp. was the most common in several varieties of grapevines, Madrid, Spain (González \& Tello 2010) while P. tarda was isolated from air in citrus plantations in Upper Egypt (Moubasher et al. 1971), and from the air of Assuit (Moubasher \& Moustafa 1974).

Setosphaeria (3 species) was isolated in high frequency from grapevine air and in moderate frequency from citrus air. S. rostrata was the main species, whereas $S$. monoceras was isolated from citrus air and $S$. pedicillata from grapevine air only. Setosphaeria was the most common fungus isolated from phyllosphere and phylloplane of banana plants cultivated in Upper Egypt (ElSaid 2001).

Trichoderma (9 species and 1 unidentified) was recorded in moderate frequency from citrus air and in low frequency from grapevine air. The most common species were T. harzianum and $T$. reese $i$ in citrus plantations and $T$. paracemosum in both plantations. Trichoderma was a component of the aerospora of Hong Kong (Turner 1966), and several varieties of grapevines, Madrid, Spain (González \& Tello 2010), and was recovered from Quercus and Acer phylloplanes, Czech Republic (Guimarães et al. 2011). T. harzianum is well known as a biological control agent against plant pathogenic fungi.

Mucor (4 species) was recovered in moderate frequency from citrus air, and in rare frequency from grapevine plantations. $M$. circinelloides was the most common species followed by $M$. hiemalis in both plantations. Rhizopus ( $R$. oryzae) was recorded in high frequency from grapevine air and in moderate frequency from citrus air. Humicola (H. fuscoatra, H. grisea, and Humicola sp.) was isolated in high frequency from citrus air and in low frequency from grapevine air. H. fuscoatra was more common than H. grisea in both plantations. Stachybotrys (S. chartarum and synnematous species of Stachybotrys) was recorded in rare frequency from the air of grapevine while in low frequency from citrus air.

Nigrospora oryzae and $N$. sphaerica were identified in low frequency from the air of both plantations, however the former was more frequent than the later. Nigrospora is a common component of the aerospora in Jamaican banana plantations (Meredith 1961), Nigeria (Cammack 1955). Also, Nigrospora, Curvularia, Pithomyces, Phoma, Epicoccum and Neurospora were the prevalent fungal genera recorded among the aerospora at Ibadan, Nigeria (Ogunlana 1975).

Acremonium (9 species and 1 unidentified) was isolated in low frequency from grapevine air and in rare frequency from citrus air. A. strictum was the most common species followed by $A$. fusidioides and A. hyalinulum. Acremonium was isolated from several varieties of grapevines, Madrid, Spain (González \& Tello 2010). 
Microascus (M. brevicaulis and M. manginii, teleomorphs of Scopulariopsis. brevicaulis and $S$. candida respectively) was isolated in low frequency from citrus air and in rare frequency from grapevine air. The two species were isolated from Egyptian soil (Moubasher 1993).

Of the 15 yeast genera (represented by 26 species) recovered, Cryptoccocus (4 species; $C$. albidus, $C$. carnescence, $C$. flavescens and C. laurentii) and Rhodotorula (3 species) were the most common, but Debaryomyces (2 species) and Sporidiobolus (S. ruineniae) were less common in the air of both plantations. Some yeast genera were recovered only from the air of citrus plantations (Ambrosiozyma, Candida, Geotrichum, Hanseniaspora, Rhodosporidium and Melanopsichium) while Sporobolomyces and Trichosporon from grapevine plantations only. The dominance of Cryptococcus albidus and Rhodotorula mucilaginosa in the air was reported by Di Menna (1955); Voros-Felkai (1966, 1967); Al-Doory (1967) and Haridy (1992). Debaryomyces hansenii was isolated from the air of El-Minia city, Egypt (Haridy 1992) and Candida sp. was recorded in the air of tea plantations of Barak Valley, Assam, India (Dutta et al. 2010).

The present study reveals that the dematiaceous fungi outnumbered the hyaline ones and Basidiomyceteous yeasts were dominant over ascomyceteous yeasts.

\section{References}

Abdel Hameed AA, Khoder MI, Yuosra S, Osman AM, Ghanem S. 2009 - Diurnal distribution of airborne bacteria and fungi in the atmosphere of Helwan area, Egypt. Science of the Total Environment 407(24), 6217-6222.

Abdel-Hafez SI 1984 - Survey of air-borne fungus spores at Taif, Saudi Arabia. Mycopathologia $88,39-44$.

Abdel-Hafez SI, Shoreit A, Abdel-Hafez IA, EL-Maghraby OM 1986 - Mycoflora and mycotoxin producing fungi of air-dust particles from Egypt - Mycopathologia 93, 25-32.

Abdul Wahid OA, Moustafa AW, Moustafa AM 1996 - Fungal population in the atmosphere of Ismailia City. Aerobiologia 12, 249-255.

Abu-Dieyeh MH, Barham R, Abu-Elteen K, Al-Rashidi R, Shaheen I 2010 - Seasonal variation of fungal spore populations in the atmosphere of Zarqa area, Jordan. Aerobiologia 26(4), 263276

Abu-El-Souod SM 1974 - Studies on fungus-air-spora in Egypt. Ph. D. Thesis, Faulty of Science, Assiut University.

Ahire PP, Kadam VB, Patel SI 2010 - Atmospheric concentration and seasonal variation in the smut spores at Nashik, Maharashtra, India. Plant Archives 10(2), 963-964.

Al-Doory Y 1967 - Further studies of the fungal flora of the air in San Antonio, Texas. Journal of Allergy 40(3), 145-150.

Al-Doory Y 1980 - Laboratory Medical Mycology, pp. 240, 357-367. Lea Febiger Philadelphia Kimpton Publishers, London.

Alhubaishi AAA, Abd-Kader MIA 1991 - Phyllosphere and phylloplane fungi of qat in Sana'a, Yemen Arab Republic. Journal of Basic Microbiology 31, 83-89.

Al-Qura'n S 2008 - Analysis of airborne pollen fall in Tafileh, Jordan, 2002-2003. World Applied Sciences Journal 4(5), 730-735.

Al-Subai AA 2002 - Air-borne fungi at Doha, Qatar. Aerobiologia 18(3-4), 175-183.

Amiri A, Bompeix G 2005 - Diversity and population dynamics of Penicillium spp. on apples in pre- and postharvest environments: consequences for decay development. Plant Pathology 54: 74-81.

Asan A, Ilhan S, Sen B, Erkara I, Filik C, Cabuk A 2004 - Airborne fungi and actinomycetes concentrations in the air of Eskisehir City (Turkey). Indoor Built Environment 13, 63-74.

Bagwan NB 2011 - Aflatoxin B1 contamination in papaya fruits (Carica papaya L.) during post harvest pathogenesis. Indian Phytopathology 64(1), 48-50.

Barkai-Gollan RR 1961 - Air-born fungi in packing houses for citrus fruits. Bulletin of the Research Council, Israel D 10, 135-141. 
Barnett JA, Payne RW, Yarrow D 2000 - Yeasts: characteristics and identification, $3 \mathrm{r}^{\mathrm{d}}$ ed. Cambridge University Press, Cambridge, England.

Ben-Meir-Gluack Y 1952 - Fungi isolated from the air of groves and packing sheds and from the skin of Shamouti oranges. Ktavim 2-3, 135-140.

Bhati SH, Gaur RD 1979 - Studies on aerobiology-atmospheric fungal spores. New Phytologist 82, 519-527.

Booth C 1971 - The genus Fusarium. Commonwealth Mycological Institute, Kew, UK,

Cammacrk H 1955 - Seasonal changes in three common constituents of the air spora in southern Nigeria. Nature 176: 1270.

Crous PW, Braun U, Schubert K, Groenewald JZ 2007 - The genus Cladosporium and similar dematiaceous hyphomycetes. Studies in Mycology 58, 1-253.

De Beer ZW, Begerow D, Bauer R, Pegg GS, Crous PW, Wingfield MJ 2006 - Phylogeny of the Quambalariaceae fam. nov., including important Eucalyptus pathogens in South Africa and Australia. Studies in Mycology 55: 289-298.

De Hoog GS de, Guarro J, Gene J, Figueras MJ 2000 - Atlas of clinical fungi. Centraalbureau voor Schimmelcultures/ Universitat Rovira i Virgili, pp. 1126.

De Seifert K, Morgan-Jones, Gams W, Kendrick B 2011 - for the genera of hyphomycetes.CBSKNAW Fungal Biodiversity Centre, Utrecht, The Netherlands, pp. 997.

Desnos-Ollivier M, Ragon M, Robert V, Raoux D, Gantier JC, and Dromer F 2008 Debaryomyces hansenii (Candida famata), a rare human fungal pathogen often misidentified as Pichia guilliermondii (Candida guilliermondii). Journal of Clinical Microbiology 46(10), 3237-3242.

Di Menna ME 1955 - A quantitative study of air-borne fungus spores in Dunedin, New Zealand. Transactions of the British Mycological Society 38(2), 119-129.

Díaz GA, Torres R, Vega M, Latorre BA 2009 - Ochratoxigenic Aspergillus species on grapes from Chilean vineyards and Aspergillus threshold levels on grapes. International Journal of Food Microbiology 133, 195-199.

Domsch KH, Gams W, Anderson TH 2007 - Compendium of soil fungi. 2nd edition, IHW-Verlag, Eching.

Dutta S, Dutta BK, Nath PK 2010 - Comparative study of the air, phyllosphere and soil mycoflora of the tea plantation area of Cachar District, Assam. Assam University Journal of Science and Technology 5(I), 89-94.

El-Essawy AA, Abd El-Kader MIA, Abou El-Hawa ME, Aly ASE 1992 - Studies on mycoflora of air of Sana'a Governorate "Yemen Republic'. Egyptian Journal of Applied Science 7, $607-616$.

El-Helaly AF, Ibrahim IA, Assawah MW, Elarosi HM, Abo-El-Dahab M K, Michail SH, Abd-ElRehim MA, Wasfy EH, El-Goorany MA 1966 - General survey of plant diseases and pathogenic organisms in the U. A. E. (Egypt) until 1965. Alexanderia Journal of Agricultural Research, Faculty of Agrculture, Alexandria University Press, Alexandria.

Ellis MB 1971 - Dematiaceous Hyphomycetes. Commonwealth Mycological Institute, Kew, Surrey, England, 608 pp.

Ellis MB 1976 - More Dematiaceous Hyphomycetes. Commonwealth Mycological Institute, Kew, Surrey, England, $481 \mathrm{pp}$.

El-Morsy ES 2006 - Preliminary survey of indoor and outdoor airborne microfungi at coastal buildings in Egypt. Aerobiologia 22, 197-210.

El-Said AH, Abdel-Hafez SI 1995 - Seasonal variations of airborne fungi above banana fields in Qena, Upper Egypt. Cryptogamie Mycologie 16, 101-109.

El-Said AHM 2001 - Phyllosphere and phylloplane fungi of banana cultivated in Upper Egypt and their cellulolytic ability. Mycobiology 29(4): 210-217

El-Sherbeny GA 1987 - Survey for pathogenic microorganisms at Zagazig area, Ph.D. thesis, Faculty of Science, Zagazig University 
Fell JW, Boekhout T, Fonseca A, Scorzetti G, Statzell-Tallman A 2000 - Biodiversity and systematics of basidiomycetous yeasts as determined by large-subunit rDNA D1/D2 domain sequence analysis. International Journal of Systematic and Evolutionary Microbiology 50(3), 1351-1371.

Fell JW, Scorzetti G, Statzell-Tallman A, Pinel N, Yarrow D 2002 - Recognition of the basidiomycetous yeast Sporobolomyces ruberrimus sp. nov. as a distinct species based on molecular and morphological analyses. FEMS Yeast Research 1(4), 265-270.

González V, Tello ML 2010 - The endophytic mycota associated with Vitis vinifera in central Spain. Fungal Diversity 47(1), 29-42.

Gräfenhan T, Schroers HJ, Nirenberg HI, Seifert KA 2011 - An overview of the taxonomy, phylogeny and typification of some nectriaceous fungi classifed in Cosmospora, Acremonium, Fusarium, Stilbella and Volutella. Studies in Mycology 68, 79-113.

Gregory PH 1973 - The Microbiology of the atmosphere. $2^{\text {nd }}$ ed., Aylesbury, Bucks, Leonard Hill, London.

Guimarães JB, Chambel L, Melzoch K, Pereira P, Tenreiro R 2011 - Cladosporium sp. from phyloplane: a diversity evaluation on a continental ecosystem. Mycosphere 2(3), 191-201.

Haridy MSA 1992 - A survey of yeasts found in the air of El-Minia city, Egypt. The Korean Journal of Mycology 20, 269-272.

Hasnain SM, Fatima K, Al-Frayh A, Al-Sedairy S 2005 - Prevalence of airborne basidiospores in three coastal cities of Saudi Arabia. Aerobiologia 21, 139-145.

Hasnain SM, Newhook FJ, Wilson JD, Corbin JB, 1984 - First report of Gnrzodcrnm allergenicity in Auckland, New Zealand. New Zealand Journal of Science 27, 261-267.

Hasnain SM, Wilson JD, Newhook FJ 1985a - Fungal allergy and respiratory diseascs. New Zealand Medical Journal 98, 342-346.

Hasnain SM, Wilson JD, Newhook FJ, 1985b - Allergy to basidiospores: Immunological study. New Zealand Medical Journal 98, 393-396.

Hedayati M, Mayahi S, Aghili R, Goharimoghadam K 2005 - Airborne fungi in indoor and outdoor of asthmatic patients' home, living in the city of Sari. Iranian Journal of Allergy, Asthma and Immunology 4(4), 189-191.

Ismail MA, Abdel-Hafez SII, Moharram AM 2002 - Aeromycobiota of western desert of Egypt. African Journal of Science and Technology 3(1): 1-9.

Ismail MA, Chebon SK, Rebecca Nakamya 1999 - Preliminary surveys of outdoor and indoor aeromycobiota in Uganda. Mycopathologia 148, 41-51.

Khan ZU, Khan M, Chandy R, Sharma PN 1999 - Aspergillus and other moulds in the air of Kuwait. Mycopathologia 146, 25-32.

King DA, Hocking AD, Pitt JI 1979 - Dichloran-rose bengal medium for enumeration and isolation of molds from foods. Applied and Environmental Microbiology 37, 959-964.

Lacey J 1981 - Aerobiology of conidial fungi. In G. T.Cole \& B. Kendrick (Eds.), Biology of conidial fungi (1: 273-416). New York: Academic.

Leslie JF, Summerell BA 2006 - Fusarium laboratory workshops- A recent history. Mycotoxin Research 22(2), 73-74.

Levetin E, Dorsey K 2006 - Contribution of leaf surface fungi to the air spora. Aerobiologia 22, 312.

Lohare S, Kamble R, Lakde H, Nagpurne VS 2009 - Air spora over tomato field at Udgir. Shodh, Samiksha aur Mulyankan (International Research Journal) 2(6): 806-807.

Mallaiah KV, Rao AS 1990 - Air spora of groundnut fields. Proc. Indian Acad. Scl. (Plant Sci.) 89(4), 289-481.

Marincowitz S, Gryzenhout M, Wingfield MJ 2010 - New and rare coelomycetes with appendagebearing conidia from Pondoland, South Africa. Mycotaxon 111, 309-322.

Mazen MB 1973 - Ecological studies on cellulose-decomposing fungi in Egypt. Ph.D. Thesis, Faculty of Science, Assuit University 
McCune B, Mefford MJ 1999 - Multivariate analysis of ecological data, Version 4. MJM Software Design, Gleneden Beach, Oregon, USA, pp. 237.

Meredith DS 1961 - Atmospheric content of Nigrospora spores in Jamaican banana plantations. Journal of General Microbiology 26, 343--349.

Molnár O, Prillinger H 2005 - Analysis of yeast isolates related to Metschnikowia pulcherrima using the partial sequences of the large subunit rDNA and the actin gene; description of Metschnikowia andauensis sp. nov. Systematic and Applied Microbiology 28, 717-726.

Moretti A, Fukushima K, Takizawa K, Suzuki M, Vidotto V, Cannizzo FT, Boncio L, Bollo E 2007 - First report of oral colonization by Debaryomyces nepalensis in a dog. Mycopathologia 164, 189-192.

Moubasher AH 1993 - Soil fungi in Qatar and other Arab countries. The Scientific and Applied Research Center, University of Qatar, Doha, pp. 566.

Moubasher AH 1995 - Soil fungi are an active partner of our ecosystem: Their biodiversity and activities should appreciated. Qatar Univ Sci J 15(1), 239-247.

Moubasher AH, Moustafa AF 1974 - Air-borne fungi at Assiut, Egypt. Egyptian Journal of Botany $17,135-149$.

Moubasher AH, Zeinab Soliman 2011 - Aspergillus assiutensis, a new species from the air of grapevine plantation, Egypt. Journal of Basic \& Applied Mycology, Egypt 2, 83-90.

Moubasher AH, Elnaghy MA, Abdel-Fatah HM 1971 - Citrus plantation fungi in Upper Egypt. Transactions of the British Mycological Society 57(2), 289-294.

Moubasher AH, Abdel-Fattah, HM, Swelim MA 1981 - Studies on air-borne fungi at Qena. I. Seasonal fluctuations. Zeitschrift fur Algemeine Mikrobiologie 21, 247-253.

Moubasher AH, Abdel-Fattah HM, Swelim MA 1982 - Studies on air-borne fungi at Qena. IV. Effect of wind velocity in total counts. Mycopathologia 80, 39-42.

Moubasher AH, Abdel-Sater MA, Zeinab Soliman 2013 - Contribution to the mycobiota of Egypt: Emericella stella-maris Zalar, Frisvad \& Samson 2008, a new record to Egypt. Journal of Basic and Applied Mycology (Egypt) 4, 85--87.

Moustafa AF 1975 - Osmophilous fungi in the salt marshes of Kuwait. Canadian Journal of Microbiology 21, 1573-1580.

Moustafa AF, Kamel SM 1976 - A study of fungal populations in the atmosphere of Kuwait. Mycopathologia 59, 29-35.

Naim MS 1967 - Contribution to the knowledge of soil fungi in Libya. II. Fungus flora under citrus trees in Libya. Mycopathologyia et Mycologia Applicata 31, 300-304.

Nourian AA, Badali H, Khodaverdi M, Hamzehei H, Mohseni S 2007 - Airborne mycoflora of Zanjan-Iran. International Journal of Agriculture \& Biology 9(4), 628-630.

Ogunlana EO 1975 - Fungal air spora at Ibadan, Nigeria. Applied Microbiology 29(4), 458-463.

Oliveira M, Ribeiro H, Abreu I 2005 - Annual variation of fungal spores in atmosphere of Porto. Annals Agricultural Environmental Medicine 12, 309-315.

Õzkara A, Ocak I, Korcan S, Konuk M 2007 - Determination of fungal air spora in Afyonkarahisar, Turkey. Mycotaxon 102, 199-202.

Panda T, Panda B, Mishra N 2009 - Seasonal Incidence of air borne fungi in coastal belt of Orissa. Journal of Human Ecology 26(3), 205-207.

Peterson SW, Jurjevic Z, Bills GF, Stchigel AM, Guarro J, Vega FE 2010 - Genus Hamigera, six new species and multilocus DNA sequence based phylogeny. Mycologia 102(4), 847--864.

Pitt J I 1979 - The genus Penicillium, Academic Press, London, 635 pp.

Rees RG 1964 - The air spora of Brisbane. Australian Journal of Botany 12 (2), 185-204.

Samson RA, Frisvad JC 2004 - Penicillium subgenus Penicillium: new taxonomic schemes, mycotoxin and other extrolites. Studies in Mycology 49, 1-260.

Samson RA, Varga J 2007 - Aspergillus systematics in the genomic era. Studies in Mycology 59, 1-206. 
Sanchez-Hervas M, Gil JV, Bisbal F, Ramon D, Martinez-Culebras PV 2008 - Mycobiota and mycotoxin producing fungi from cocoa beans. International Journal of Food Microbiology 125: 336-340.

Sarica S, Asan A, Tatman-Otkun M, Ture M 2002 - Monitoring indoor airborne fungi and bacteria in the different areas of Trakya University Hospital (Edirne-Turkey). Indoor Built Environment 11, 285-292.

Schroers HJ 2001 - A monograph of Bionecteria (Ascomycota, Hypocreales, Bionecteriaceae) and its Clonostachys anamorphs. Studies in Mycology 46, 1-214.

Scorzetti G, Fell JW, Fonseca A, Statzell-Tallman A 2002 - Systematics of basidiomycetous yeasts: a comparison of large subunit D1/D2 and internal transcribed spacer rDNA regions. FEMS Yeast Research 2(4), 495-517.

Sen B, Asan A 2001 - Airborne fungi on vegetable growing areas of Edirne, Turkey. Aerobiologia 17(1), 69-75.

Shaheen I 1992 - Aeromycology of Amman area, Jordan. Grana 31, 223-228.

Simmons EG 2007 - Alternaria: An identification manual. CBS Fungal Biodiversity Centre, Utrecht, Netherlands.

Smith NI, Dawson VT 1944 - The Bacteriostatic Action of Rose Bengal in Media Used for the Plate Counts of Fungi. Soil Science 58, 467-471.

Soliman ZSM 2012 - Studies on yeast and filamentous fungi in citrus and grapevine plantations. M. Sc. Thesis, Faculty of Science, Assiut University, Assiut, Egypt.

Stoll M, Begerow D, Oberwinkler F 2005 - Molecular phylogeny of Ustilago, Sporisorium, and related taxa based on combined analyses of rDNA sequences. Mycological Research 109(3), 342-356.

Suh S-O, Zhang N, Nguyen N, Gross S, Blackwell M 2008 - Lab Manual for yeast study. Mycology Lab., Louisiana State University, pp: 3-38.

Suryanarayanan TS, Govindarajulu MB, Thirumalai E., and Reddy M. S. (2011) - Nicholas P. MoneyAgni's fungi: heat-resistant spores from the Western Ghats, southern India. Fungal Biology 115(9), 833-838.

Takahashi T 1997 - Airborne fungal colony-forming units in outdoor and indoor environments in Yokohama, Japan. Mycopathologia 139, 23-33.

Turner P D 1966 - The fungal air spora of Hong Kong as determined by the agar plate method. Transactions of the British Mycological Society 49(2), 255-267.

Valerio E, Gadanho M, Sampaio JP 2008 - Reappraisal of the Sporobolomyces roseus species complex and description of Sporidiobolus metaroseus sp. nov. International Journal of Systematic and Evolutionary Microbiology 58(3), 736-741.

Vorbs-Felkai G 1966 - Incidence of Rhodotorula species in urban air. Acta Microbiologica Academiae Scientiarum Hungaricae 13, 53-58.

Voros-Felkai G 1967 - Incidence of Cryptococcus species in urban air. Acta Microbiologica Academiae Scientiarum Hungaricae 14, 305-308.

Wang Q-M, Jia J-H, Bai F-Y 2006 - Pseudozyma hubeiensis sp. nov. and Pseudozyma shanxiensis sp. nov., novel ustilaginomycetous anamorphic yeast species from plant leaves. International Journal of Systematic and Evolutionary Microbiology 56, 289-293.

Wickerham LJ 1951 - Taxonomy of yeasts. Tech. Bull. Nr. 1029 Dep. Agr., Washington D.C.

Yang SP, Wu ZH, Jian JC 2011 - Distribution of Marine Red Yeasts in Shrimps and the Environments of Shrimp Culture. Current Microbiology 62, 1638-1642.

Zalar P, Frisvad JC, Gunde Cimerman N, Varga J, Samson RA 2008 - Four new species of Emericella from the Mediterranean region of Europe. Mycologia, 100(5), 779-795.

Zare R, Gams W 2004 - A monograph of Verticillium section Prostrata. Rostaniha (Botanical Journal of Iran) No. 3, pp. 180. 